\title{
Oxidative Deamination of Emixustat by Human Vascular Adhesion Protein-1/Semicarbazide-Sensitive Amine Oxidase ${ }^{\S}$
}

\author{
Michael J. Reid, Russell Eyre, and Terry Podoll
}

Acucela Inc., Seattle, Washington (M.J.R.); MavuPharma, Kirkland, Washington (R.E.); and IV-PO, LLC, Seattle, Washington (T.P.)

Received December 13, 2018; accepted January 28, 2019

\begin{abstract}
Emixustat potently inhibits the visual cycle isomerase retinal pigment epithelium protein 65 (RPE65) to reduce the accumulation of toxic bisretinoid by-products that lead to various retinopathies. Orally administered emixustat is cleared rapidly from the plasma, with little excreted unchanged. The hydroxypropylamine moiety that is critical in emixustat's inhibition of RPE65 is oxidatively deaminated to three major carboxylic acid metabolites that appear rapidly in plasma. These metabolites greatly exceed the plasma concentrations of emixustat and demonstrate formation-rate-limited metabolite kinetics. This study investigated in vitro deamination of emixustat in human vascular membrane fractions, plasma, and recombinant human vascular adhesion protein-1 (VAP-1), demonstrating single-enzyme kinetics for the formation of a stable aldehyde intermediate
\end{abstract}

\section{Introduction}

Emixustat (Chemical Abstracts Service number 1141777-14-1, ACU4429 ) is a potent orally administered small-molecule inhibitor of retinal pigment epithelium 65 (RPE65) under clinical development by Acucela Inc. for the potential treatment of various retinopathies, including Stargardt disease and proliferative diabetic retinopathy. RPE65 is a key isomerohydrolase enzyme in the pathway that regenerates the visual chromophore (11-cis retinal) in vertebrate retinal tissue. Emixustat decreases the levels of vitamin A derivatives (11-cis- and all-trans-retinal) and the accumulation of toxic lipid-retinoid by-products (e.g., $N$-retinylidene- $N$ retinylethanolamine and lipofuscin) that result in dysfunction and eventual death of the specialized photoreceptor-containing cells of the retina (Bavik et al., 2015). While important advances have been achieved in the treatment of the wet form of age-related macular degeneration (AMD; Gower et al., 2016) using injectable vascular endothelial growth factor inhibitors, oral administration for retinal diseases remains an attractive objective given the infection risks and frequent office visits associated with intravitreal injection (Awh, 2016).

This work was supported by Acucela Inc., and has not been previously presented.

https://doi.org/10.1124/dmd.118.085811

SThis article has supplemental material available at dmd.aspetjournals.org.
(ACU-5201) in all in vitro systems. The in vitro systems used herein established sequential formation of the major metabolites with addition of assay components for aldehyde dehydrogenase and cytochrome P450. Reaction phenotyping experiments using selective chemical inhibitors and recombinant enzymes of monoamine oxidase, VAP-1, and lysyl oxidase showed that only VAP-1 deaminated emixustat. In individually derived human vascular membranes from umbilical cord and aorta, rates of emixustat deamination were highly correlated to VAP-1 marker substrate activity (benzylamine) and VAP1 levels measured by enzyme-linked immunosorbent assay. In donormatched plasma samples, soluble VAP-1 activity and levels were lower than in aorta membranes. A variety of potential comedications did not strongly inhibit emixustat deamination in vitro.

Emixustat was designed to treat the retina following oral administration by focusing on potent and durable pharmacologic activity against RPE65 while displaying rapid systemic elimination. Preclinical demonstration of these traits translated well to clinical application in a phase 1 study wherein dose-dependent effects were measured in the retina more than 24 hours after dose, at a time when emixustat levels were very low due to its rapid elimination (4- to 6-hour half-life in plasma; Kubota et al., 2012). Emixustat absorption was also rapid, with $\mathrm{T}_{\max }$ values ranging from 3 to 5 hours, while no accumulation was observed upon repeated-dose administration (Kubota et al., 2014). A human absorption, distribution, metabolism, and excretion (ADME) study characterized three major (i.e., $\geq 10 \%$ of total systemic ${ }^{14} \mathrm{C}$ exposure) circulating metabolites: ACU-5116, ACU 5124, and ACU-5149, accounting for $11.5 \%, 29.0 \%$, and $10.6 \%$ of the total area under the plasma drug concentration-time curve (AUC) of circulating radioactivity (Fitzsimmons et al., 2018). In this study, $>90 \%$ of emixustat doserelated radioactive material was excreted via the urine as metabolites, similar to nonclinical species (data on file).

Preclinical in vivo metabolism of emixustat was very similar across rats, dogs, and monkeys (Podoll et al., 2013). The three major metabolites are carboxylic acids that are products of oxidative deamination of the primary amine of emixustat (Fitzsimmons et al., 2018). Two of the three metabolites, ACU-5124 and ACU-5149, were secondary metabolites that had also been differentially hydroxylated in the cyclohexyl

ABBREVIATIONS: ACN, acetonitrile; ADME, Absorption, Distribution, Metabolism and Excretion; AMD, age-related macular degeneration; AUC, area under the plasma drug concentration-time curve; BA, benzylamine; BAL, benzaldehyde; BALDNP, benzaldehyde dinitrophenylhydrazone; $\mathrm{CPQ}$, carboxyprimaquine; DDI, drug-drug interaction; DEABAL, 4-diethylamino-benzaldehyde; DNPH, 2,4-dinitrophenylhydrazine; ELISA, enzymelinked immunosorbent assay; hAM, human aorta membrane; hLM, human liver microsome; hP, human plasma; HPLC, high-performance liquid chromatography; hUCM, human umbilical cord membrane; LC-MS/MS, liquid chromatography-tandem mass spectrometry; LOX, lysyl oxidase; MAO, monoamine oxidase; MPA, mobile phase A; P450, cytochrome P450; PK, pharmacokinetic; PQ, primaquine; pTALDNP, p-tolualdehyde dinitrophenylhydrazone; rhMAO, recombinant human MAO; rhVAP-1, recombinant human VAP-1; RPE65, retinal pigment epithelium protein 65 ; SSAO, semicarbazide-sensitive amine oxidase; VAP-1, vascular adhesion protein-1. 
ring. In human hepatocytes, primary hydroxylation to the cyclohexanols dominated the product formation observed, averaging $26 \%$ of radioactivity, and only 4\%-9\% observed as the oxidatively deaminated carboxylic acids (Fitzsimmons et al., 2018). In human liver microsomes, no oxidative deamination was observed, and NADPH-dependent turnover of $\left[{ }^{14} \mathrm{C}\right]$ emixustat was low, yet CYP2D6 and CYP3A4 were identified as capable of catalyzing the hydroxylation of emixustat in vitro when using the cDNA-expressed cytochrome P450 (P450) isoforms (data on file). Other extrahepatic, human in vitro systems were then used to characterize the predominant oxidative deamination reaction observed in the human ADME clinical trial.

Initial studies focused on monoamine oxidases (MAOs), yet emixustat was not a substrate for MAO (data on file), so alternative methods were sought to characterize its oxidative deamination. The first evidence of vascular adhesion protein-1/semicarbazide-sensitive amine oxidase (VAP-1/SSAO, from here forward referred to as VAP-1) involvement in the metabolism of emixustat was revealed during a cross-species plasma protein binding study (Fitzsimmons et al., 2018; additional nonclinical species data on file). Plasma stability assessments demonstrated interspecies differences that were consistent with the highest reported soluble plasma deamination activity being observed in pigs and dogs (Schwelberger, 2007). Prior studies have used human umbilical artery microsomes to demonstrate the involvement of VAP-1 in the metabolism of tresperimus (Claud et al., 2002). Since then, potent, selective VAP-1 inhibitors have been developed (Salter-Cid et al., 2005), and recombinant VAP-1 has become commercially available. The results of human in vitro reaction phenotyping for the oxidative deamination of emixustat are described here. Since emixustat was targeting retinopathies that are more prevalent in adults aged 65 years and above (e.g., AMD), and this population can have inflammatory diseases impacting VAP-1 (Salmi et al., 1993; Pannecoeck et al., 2015), human aorta tissue was obtained from elderly donors to characterize emixustat oxidative deamination in this patient population. Once established, the in vitro model was used to assess the impact of potential VAP-1 inhibitors on emixustat and the impact of emixustat on the VAP-1-catalyzed oxidative deamination of the model substrate, benzylamine (BA).

\section{Materials and Methods}

Materials. Emixustat hydrochloride ( $99.7 \%$ chemical purity) was manufactured by IRIX Pharmaceuticals (Florence, SC). ACU-4861 [(1R,4r)-4-((3-((R)-3amino-1-hydroxypropyl)phenoxy)methyl)cyclohexan-1-ol], ACU-5116 [(R)-3(3-(cyclohexylmethoxy)phenyl)-3-hydroxypropanoic acid], ACU-5124 [(R)-3hydroxy-3-(3-(((1r,4R)-4-hydroxycyclohexyl)methoxy)phenyl)propanoic acid], ACU-5141 (d11-ACU-5116), ACU-5142 (d3-ACU-5124), and ACU-5201 [(E)-3-(3-(cyclohexylmethoxy)phenyl)acrylaldehyde] were prepared by Acucela (Bothell, WA) as described previously (Fitzsimmons et al., 2018; Supplemental Data). LJP-1207 [ $N^{\prime}$-(2-phenyl-allyl)-hydrazine hydrochloride] was also prepared and characterized by Acucela according to previously published methods (SalterCid et al., 2005). Acetic acid, methanol [high-performance liquid chromatography (HPLC) grade], phosphate-buffered saline, sodium bicarbonate, and water (HPLC grade) were obtained from Fisher Scientific USA (Pittsburg, PA). All other chemicals and reagents were obtained from Sigma-Aldrich (St. Louis, MO).

Recombinant human VAP-1, the enzyme-linked immunosorbent assay (ELISA) kit for human VAP-1, anti-human VAP-1 antibody, as well as recombinant human lysyl oxidase homolog 2 (LOXL2) were obtained from R\&D Systems (Minneapolis, MN). Anti-mouse horseradish peroxidase antibody was obtained from Promega (Madison, WI). Human liver microsomes (hLMs; UltraPool HLM 150 Mixed Gender, lot \#38290) were obtained from BD Biosciences (Woburn, MA). Human umbilical cord membranes (hUCMs) and elderly human aorta membranes (hAMs) with matching heparinized plasma samples from the elderly humans were prepared under contract by BioIVT (Baltimore, MD). Twenty individual lots each of hUCM and hAM and 18 donormatched (to hAM) individual plasma samples were provided to assess interindividual variability of VAP-1 activity. An eight-donor pool of hUCM and a 20-donor pool of hAM were also provided to determine the kinetics of VAP-1 activity. Aorta tissues were obtained from individuals who were at least 65 years of age with the following demographics: 11 male and nine female, three African American, four nonwhite Hispanic, and 13 Caucasian, with body mass index ranging from 16.9 to 43 . Eight individuals were identified as having diabetes and 17 had cardiovascular complications and/or hypertension.

Sequential Oxidative Metabolism of Emixustat in Human Tissue. Pooled hUCM, NAD+, emixustat, and its hydroxycyclohexyl metabolite (ACU-4861) were prepared in $10 \mathrm{mM}$ sodium bicarbonate buffer $(\mathrm{pH} 7.4)$ and kept on wet ice until being added to incubations. Final incubation volumes of hUCM reactions were $0.2 \mathrm{ml}$ at a final protein concentration of $0.1 \mathrm{mg} / \mathrm{ml}$ with final concentrations of substrates (100 $\mu \mathrm{M}$ emixustat or ACU-4861) and the cofactor $\mathrm{NAD}^{+}(1 \mathrm{mM})$. Reactions were started by the addition of hUCM immediately followed by the addition of $\mathrm{NAD}^{+}$and stopped after 20 minutes with the addition of $0.2 \mathrm{ml}$ of acetonitrile (ACN), vortexed briefly, and centrifuged at $13,000 \mathrm{~g}$ for 10 minutes to precipitate proteins. Supernatants were filtered through $0.2-\mu \mathrm{m}$ polypropylene filter membranes (Captiva; Agilent) and loaded onto 96-well plates.

hLMs, NADPH, and the deaminated carboxylic acid metabolite (ACU-5116) were prepared in $10 \mathrm{mM}$ potassium phosphate buffer $(\mathrm{pH} 7.4)$ and kept on wet ice until being added to incubations. Final incubation volumes of hLM reactions were $0.2 \mathrm{ml}$, final microsomal protein concentrations were $1.0 \mathrm{mg} / \mathrm{ml}$, final ACU-5116 concentrations were $100 \mu \mathrm{M}$, and final concentrations of NADPH were $1 \mathrm{mM}$. Reactions were started by the addition of NADPH to hLM incubations preincubated at $37^{\circ} \mathrm{C}$ for 3 minutes. Reactions were stopped after 20 minutes with the addition of $0.1 \mathrm{ml}$ of $\mathrm{ACN}$, vortexed briefly, and centrifuged at $13,000 \mathrm{~g}$ for 10 minutes to precipitate proteins. Supernatants were filtered through $0.2-\mu \mathrm{m}$ polypropylene filter membranes (Captiva; Agilent) and loaded onto 96-well plates.

In Vitro Metabolism of Emixustat in Human Sources of VAP-1. In vitro metabolism of emixustat by human sources of VAP-1 was determined by measuring the amount of its stable aldehyde product (ACU-5201) formed per minute per milligram of protein (or milliliter of plasma) in triplicate. Final incubation volumes were $0.1 \mathrm{ml}$, and the amount of VAP-1 source and incubation time were linearly optimized. Pooled and individual lots of hUCM or hAM were prepared at a final protein concentration of $0.1 \mathrm{mg} / \mathrm{ml}$ and recombinant human VAP-1 was prepared at a final protein concentration of $0.01 \mathrm{mg} / \mathrm{ml}$, whereas human plasma $(\mathrm{hP})$ was assayed volumetrically $(0.05 \mathrm{ml})$ in $10 \mathrm{mM}$ sodium bicarbonate buffer, $\mathrm{pH} 7.4\left(\mathrm{NaHCO}_{3}\right.$ buffer). Stock solutions of emixustat and inhibitors were also prepared in $\mathrm{NaHCO}_{3}$ buffer and were preincubated at $37^{\circ} \mathrm{C}$ together (when inhibition was tested) prior to adding the enzyme source. Human sources of VAP-1 were kept on dry ice until thawed at $37^{\circ} \mathrm{C}$ prior to diluting with $\mathrm{NaHCO}_{3}$ buffer. When possible, timed incubations [10 minutes for hUCM, hAM, and recombinant human VAP-1 (rhVAP-1) and 30 minutes for plasma] were started immediately by the addition of the enzyme source and stopped by the addition of $0.2 \mathrm{ml}$ of ACN fortified with $10 \mathrm{ng} / \mathrm{ml}$ of 4-diethylaminobenzaldehyde (DEABAL, as an internal standard), vortexed briefly, and centrifuged at 13,000g for 10 minutes to precipitate proteins. During simultaneous incubations of tissue from several individuals, the membrane preparations were kept on wet ice until being aliquoted and brought to $37^{\circ} \mathrm{C}$ before combination with substrate. Emixustat concentrations ranged from 5.0 to $500 \mu \mathrm{M}$ when determining enzyme kinetic parameters in the various human sources of VAP-1 and between 60 and $160 \mu \mathrm{M}$ when assessing interindividual activity and inhibition. Supernatants were filtered through $0.2-\mu \mathrm{m}$ polyvinylidene fluoride membranes (Captiva; Agilent) and loaded onto 96-well plates for quantification by liquid chromatography-tandem mass spectrometry (LC-MS/MS).

LC-MS/MS Quantitation of Emixustat's Primary In Vitro Aldehyde Metabolite (ACU-5201). Although ACU-5201 has only been observed in vitro (Fitzsimmons et al., 2018), the proposed intermediate aldol (Fig. 1) has not been identified, nor could it be synthesized due to apparent instability during isolation and purification. The amount of ACU-5201 formed in VAP-1 incubations was quantitated by LC-MS/MS. ACU-5201 standard was diluted in ACN and used for preparation of calibration standard stock solutions that were added at $10 \%$ (v:v) to appropriate volumes of incubation reaction medium and acetonitrile to match the sample incubations.

Liquid chromatography (LC20AD; Shimadzu, Pleasanton, CA) separation was achieved on a Poroshell 120 Phenyl Hexyl, $2.1 \times 50-\mathrm{mm}, 2.7-\mu \mathrm{m}$ column (Agilent) using a step gradient of $10 \mathrm{mM}$ ammonium formate [mobile phase A (MPA)] and $\mathrm{ACN}$ at $0.45 \mathrm{ml} / \mathrm{min}$ as follows: 0.25 minutes $60 \% \mathrm{MPA}$, 
In vitro: vascular membranes

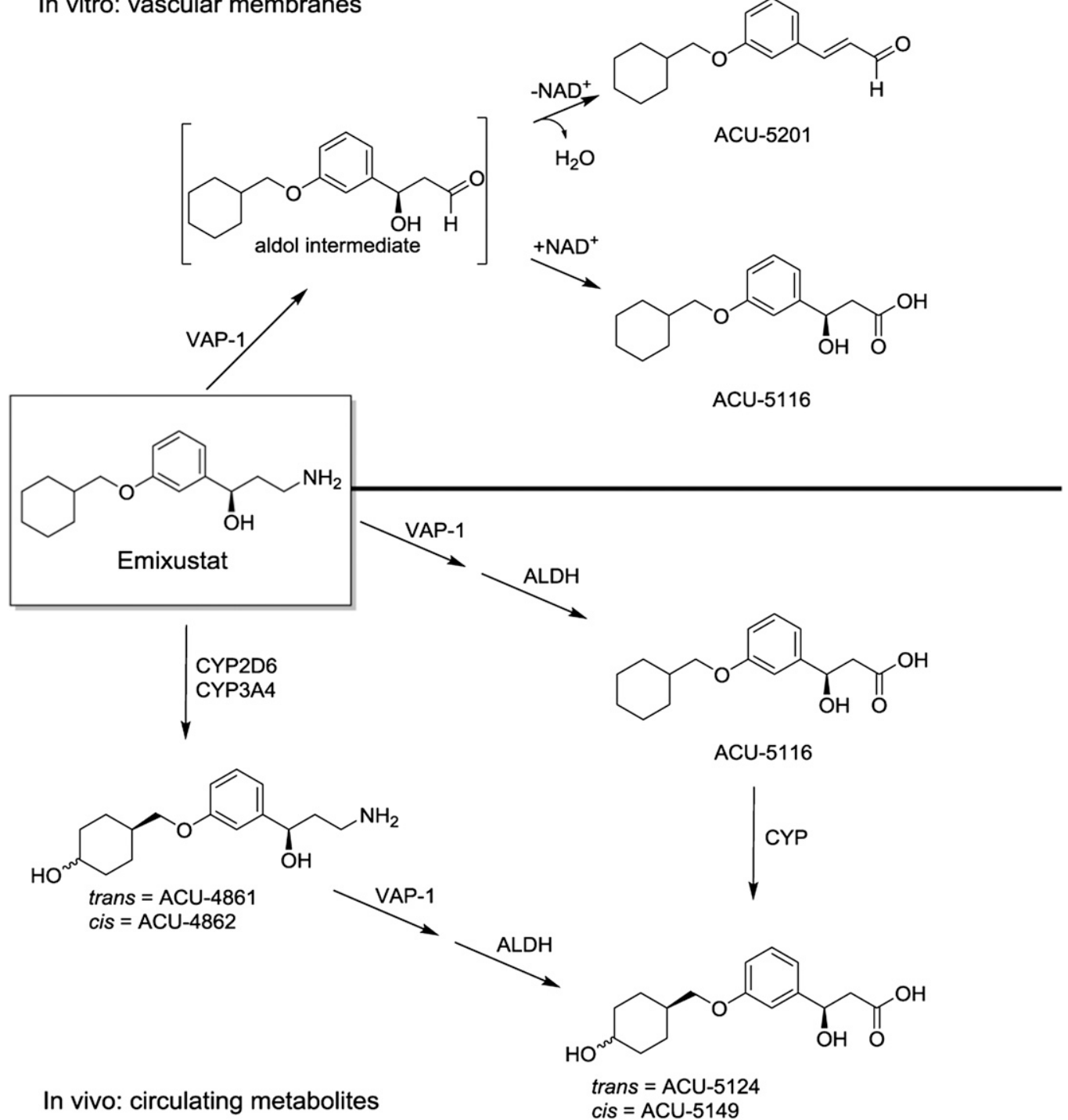

Fig. 1. Oxidative deamination scheme of emixustat to its major metabolites. The scheme above the solid bar depicts the products formed in vitro; those below the bar represent pathways leading to the major circulating metabolites observed in vivo.

0.25 minutes linear gradient to $17 \%$ MPA, 0.75 minutes $17 \%$ MPA, 0.25 minutes linear gradient to $8 \%$ MPA, then 0.5 minutes at $8 \%$ MPA before flushing with $100 \% \mathrm{ACN}$ and re-equilibrating to $60 \%$ MPA. Column temperature was set to $30^{\circ} \mathrm{C}$ while autosampler temperature was set to $10^{\circ} \mathrm{C}$. Samples $(20 \mu \mathrm{l})$ were injected with an external rinse program using $100 \% \mathrm{ACN}$.

The mass spectrometer (API5000; SCIEX, Redwood City, CA) was operated in the positive ion mode and multiple reaction monitoring scan type. The first and third quadrupoles were set to detect the positively charged molecular ions ([M $\left.+\mathrm{H}]^{+}\right)$and fragments of ACU-5201 (m/z 245.2 $\left.\rightarrow 103.1 \mathrm{Da}\right)$ and DEABAL $(\mathrm{m} / \mathrm{z}$ $178.2 \rightarrow 106.1 \mathrm{Da})$ with unit resolution in both quadrupoles. Compounddependent MS/MS parameters for quantitating ACU-5201 and DEABAL were as follows: declustering potential $=60.0$ and $175.0 \mathrm{~V}$; entrance potential $=10.0 \mathrm{~V}$; collision energy $=37.0$ and $40.0 \mathrm{~V}$; exit potential $=15.0$ and $17.0 \mathrm{~V}$, respectively. Source and gas parameters were as follows: collision gas $=8$ psi, curtain gas $=$ $11 \mathrm{psi}$, nebulizer gas $=50 \mathrm{psi}$, turbo gas $=60 \mathrm{psi}$, electrospray ionization voltage $=$ $5500 \mathrm{~V}$, and temperature $=675^{\circ} \mathrm{C}$.

In Vitro Deamination of Benzylamine in Human Sources of VAP-1. Oxidative deamination of BA was used as a biomarker of VAP-1/SSAO enzymatic activity in human sources of VAP-1 in vitro. The rate of BA deamination was determined by measuring the amount of benzaldehyde (BAL) formed per minute per milligram of protein (or milliliter of plasma) in triplicate (only duplicates for some plasma incubations). Final incubation volumes were
$0.1 \mathrm{ml}$, and the amount of VAP-1 source and incubation time were linearly optimized. Pooled and individual lots of hUCM or hAM were prepared at a final protein concentration of $0.1 \mathrm{mg} / \mathrm{ml}$ and rhVAP-1 was prepared at a final protein concentration of $0.0025 \mathrm{mg} / \mathrm{ml}$, whereas plasma was assayed volumetrically $(0.05 \mathrm{ml})$ in $\mathrm{NaHCO}_{3}$ buffer. Final reaction volumes were $0.1 \mathrm{ml}$, and timed incubations were started by the addition of the enzyme source at $37^{\circ} \mathrm{C}$. When inhibition and interindividual activity was tested, BA concentrations were $80 \mu \mathrm{M}$ for membrane and recombinant sources and $200 \mu \mathrm{M}$ for plasma. Reactions were stopped after 10 minutes for membrane and recombinant sources and after 30 minutes for plasma by the addition of $0.2 \mathrm{ml}$ of $60 / 40 \mathrm{ACN} / \mathrm{MeOH}$ fortified with $5 \mu \mathrm{M}$ internal standard $p$-tolualdehyde dinitrophenylhydrazone (pTALDNP), vortexed briefly, and centrifuged at $13,000 \mathrm{~g}$ for 10 minutes to precipitate proteins. Supernatants $(0.25 \mathrm{ml})$ were filtered through $0.2-\mu \mathrm{m}$ polyvinylidene fluoride membranes (Captiva; Agilent) and loaded onto 96-well plates, with each well containing $50 \mu \mathrm{l}$ of 2,4-dinitrophenylhydrazine (DNPH; $3 \mathrm{mg} / \mathrm{ml}$ ) solution. Derivatizations were incubated at $40^{\circ} \mathrm{C}$ for 20 minutes and stopped by the addition of $10 \mu 1$ of $25 \%$ formic acid, and then vortexed briefly prior to loading into the autosampler. Initial incubations in hUCM did not incorporate the internal standard in a reaction stop solution of acetonitrile with $2 \%$ formic acid, and these derivatizations were not stopped with formic acid. These method optimizations resulted in improved recovery of the benzaldehyde dinitrophenylhydrazone (BALDNP) by $>50 \%$ across the calibration range when 
compared with authentic BALDNP standard spiked into reaction mixtures (data not shown), affording better sensitivity to assess inhibition.

LC-MS/MS Quantitation of Derivatized Benzaldehyde. The amount of derivatized benzaldehyde (BALDNP) formed in VAP-1 incubations was quantitated by LC-MS/MS. Benzaldehyde standard was diluted in methanol to $4 \mathrm{mM}$ and used for preparation of calibration standard and quality control stock solutions. To match the sample incubations, stock solutions of BAL were added at $10 \%$ (v:v) to appropriate volumes of incubation reaction medium and internal standard solution. Calibration standards and quality controls were derivatized along with incubation samples following centrifugation according to the procedure outlined earlier.

Liquid chromatography separation was achieved on a Kinetix Biphenyl 2.5$\mu \mathrm{m}, 2 \times 30$-mm column (Phenomenex) using a step gradient of 0.15 formic acid (MPA) and $60 \% \mathrm{ACN} / 40 \% \mathrm{MeOH}$ (mobile phase $\mathrm{B}$ ) at $0.8 \mathrm{ml} / \mathrm{min}$ as follows: 0.8 minutes $34 \%$ MPA, 1.0 minute linear gradient to $2 \% \mathrm{MPA}$, then 1.0 minute $2 \%$ MPA before re-equilibrating to $34 \%$ MPA. Column temperature was set to $30^{\circ} \mathrm{C}$ while autosampler temperature was set to $6^{\circ} \mathrm{C}$. Samples $(20 \mu \mathrm{l})$ were injected with an external rinse program using $100 \% \mathrm{ACN}$.

The mass spectrometer was operated in the positive ion mode and multiple reaction monitoring scan type. The first and third quadrupoles were set to detect the positively charged molecular ions $\left([\mathrm{M}+\mathrm{H}]^{+}\right)$and fragments of BALDNP $(\mathrm{m} / \mathrm{z}$ $287.2 \rightarrow 104.1 \mathrm{Da})$ and $\mathrm{pTALDNP}(\mathrm{m} / \mathrm{z} 301.1 \rightarrow 118.3 \mathrm{Da})$ with unit resolution in both quadrupoles. Compound-dependent MS/MS parameters for quantitating BALDNP and pTALDNP were as follows: declustering potential $=70.0 \mathrm{~V}$, entrance potential $=10.0 \mathrm{~V}$, collision energy $=24.0 \mathrm{~V}$, and exit potential $=15.0 \mathrm{~V}$. Source and gas parameters were as follows: collision gas $=10$ psi, curtain gas $=$ $14 \mathrm{psi}$, nebulizer gas $=50 \mathrm{psi}$, turbo gas $=55 \mathrm{psi}$, electrospray ionization voltage $=$ $5200 \mathrm{~V}$, and temperature $=700^{\circ} \mathrm{C}$.

Quantification of VAP-1 by ELISA. Vascular adhesion protein-1 levels in membranes and plasma were quantified using the R\&D Systems Quantikine ELISA for human VAP-1. The ELISA was performed according to the instructions in the manual. The samples were diluted 1:100 with Calibrator Diluent RD6X provided by R\&D Systems. The optical density for the plate was read at 450 and $570 \mathrm{~nm}$. To account for wavelength correction, the 570-nm value was subtracted from the 450-nm value. The background value (value from well without any sample, standard, or control) was subtracted from all wells. VAP-1 concentrations (nanograms per milliliter) for all samples determined from the calibration standards were normalized to total protein concentrations determined by standard methods (Smith et al., 1985) into micrograms of VAP-1/mg of total protein.

Clinical Study Analyses. Plasma concentrations of emixustat and its three major metabolites were determined in a clinical pharmacology study titled "Pharmacokinetic and Pharmacodynamic Study of Emixustat Hydrochloride in Subjects With Geographic Atrophy Associated With Dry Age-Related Macular Degeneration" (Dugel et al., 2015) using a validated LC-MS/MS method (Podoll et al., 2018). The study was performed in accordance with the ethical principles stated in the Declaration of Helsinki; Food and Drug Administration regulation 21 Code of Federal Regulations, parts 50, 56, and 312; and the International Conference on Harmonization guidelines for good clinical practice. The protocol, advertisement, and informed consent form were reviewed and approved by the institutional review board. Written informed consent for the study was obtained from all subjects before protocol-specific procedures were carried out. Emixustat was administered orally at doses ranging from 2 to $10 \mathrm{mg}$ once daily with plasma samples collected to meet study objectives.

Data Analysis. Linear regression analysis of calibration curve data and resulting unknown sample concentration determination was performed with Analyst software (versions 1.5.1 through 1.6.2; AB Sciex) using the ratio of product to internal standard as the dependent variable. Mathematical conversion of product formation data and basic statistical calculations were performed using Microsoft Excel 2010. Michaelis-Menten and other enzyme kinetic data analyses were performed using nonlinear hyperbolic one-site binding or competition fits in GraphPad Prism software (versions 4-7; GraphPad Software, La Jolla, CA). Log values from the ELISA standards (log ng/ml VAP-1) were plotted against the corrected OD570 also using GraphPad Prism with the four-parameter logistic curve fit. Pearson and Spearman rank correlation analysis and analysis of variance of VAP-1 metabolic activities and VAP-1 levels were also performed using GraphPad Prism software. Semilog concentration-time plots for the clinical pharmacology study were also constructed using GraphPad Prism software. All noncompartmental pharmacokinetic (PK) analyses were performed using WinNonlin (version 6.3; Certara L.P., Princeton, NJ).

\section{Results}

\section{Oxidative Deamination of Emixustat}

The three major circulating metabolites observed following the dosing of $\left[{ }^{14} \mathrm{C}\right]$ emixustat during a human ADME clinical trial (ACU-5116, ACU-5124, and ACU-5149) were all carboxylic acid products of oxidative deamination of the primary amine. While the intermediate aldehydes were not observed in vivo in human plasma, ACU-5201 was identified during in vitro human plasma stability tests using $\left[{ }^{14} \mathrm{C}\right]-$ emixustat (Fitzsimmons, et al., 2018). Similar to a previous tresperimus report (Claud et al., 2001), exposure of emixustat was increased approximately 2-fold in rats following prior administration of hydralazine and semicarbazide, suggesting the rate-determining role of VAP-1 in the elimination of emixustat (data on file). The metabolic scheme shown in Fig. 1 was proposed to initiate in vitro studies to characterize the enzymology relevant to the human in vivo metabolism of emixustat. Range-finding incubations of emixustat in hUCMs, an established source of VAP-1 activity, suggested the ACU-5201 aldehyde was the predominant in vitro product. Further experiments were conducted with emixustat to confirm that ACU-5201 formation kinetic parameters could be accurately determined.

To monitor formation of the carboxylic acid metabolite, a quantitative method for ACU-5116 using synthetic reference standard and deuterium-labeled internal standard was established. Emixustat ( $50 \mu \mathrm{M} ; 80 \%$ maximal rate) was incubated at $37^{\circ} \mathrm{C}$ with hUCM at $0,0.1$, and $0.25 \mathrm{mg} / \mathrm{ml}$ total protein up to 16 minutes. Whereas levels of ACU5116 were below limit of quantitation, ACU-5201 was formed linearly with both time and protein at concentrations ranging from approximately 100 to $1000 \mathrm{ng} / \mathrm{ml}$. Although several oxidases may contribute to the rapid oxidation of the aldehyde intermediate in vivo, the role of aldehyde dehydrogenase in the conversion to the carboxylic acid was tested (Marchitti et al. 2008). Emixustat (16 $\mu \mathrm{M}$; at $\mathrm{K}_{\mathrm{M}}$, discussed later) was added to pooled hUCM $(1 \mathrm{mg} / \mathrm{ml}$ protein $)$ in the presence and absence of $\mathrm{NAD}^{+}$. High levels of ACU-5116 were observed in the presence of $\mathrm{NAD}^{+}$and were nearly the molar equivalent to the decrease in the formation of ACU-5201 when compared with the incubations without $\mathrm{NAD}^{+}$(Fig. 2A). Experiments exploring the formation route(s) of the secondary metabolite, ACU-5124, resulting from both oxidative deamination of the primary amine and hydroxylation of the cyclohexyl ring of emixustat (Fig. 1), were conducted with two primary metabolites of emixustat. To maximize potential product formation, $100 \mu \mathrm{M}$ ACU5116 was incubated with human liver microsomes $(1 \mathrm{mg} / \mathrm{ml}$ protein $)$ in the presence and absence of NADPH. NADPH-dependent formation of ACU-5124 was observed, confirming that ACU-5116 is a substrate for P450 enzymes resulting in the formation of ACU-5124. Also at a high concentration to maximize product formation, ACU-4861 (100 $\mu \mathrm{M})$, a monohydroxylated metabolite previously shown to be formed by $\mathrm{P} 450$ enzymes in human hepatocytes (Fitzsimmons et al., 2018), was incubated with pooled hUCM ( $1 \mathrm{mg} / \mathrm{ml}$ protein) for 20 minutes in the presence and absence of $\mathrm{NAD}^{+}$. $\mathrm{NAD}^{+}$-dependent formation of the carboxylic acid metabolite, ACU-5124, was observed, demonstrating that its formation could also proceed from initial hydroxylation of emixustat, followed by oxidative deamination and aldehyde oxidation of the monohydroxylated amine metabolite (Fig. 2B). Given these results, vascular membranes were used to generate kinetic parameters for the oxidative deamination of emixustat via ACU-5201 formation rates as an in vitro model for assessing the variability of VAP-1 reaction rates across a population of individually derived vascular tissues and for the 

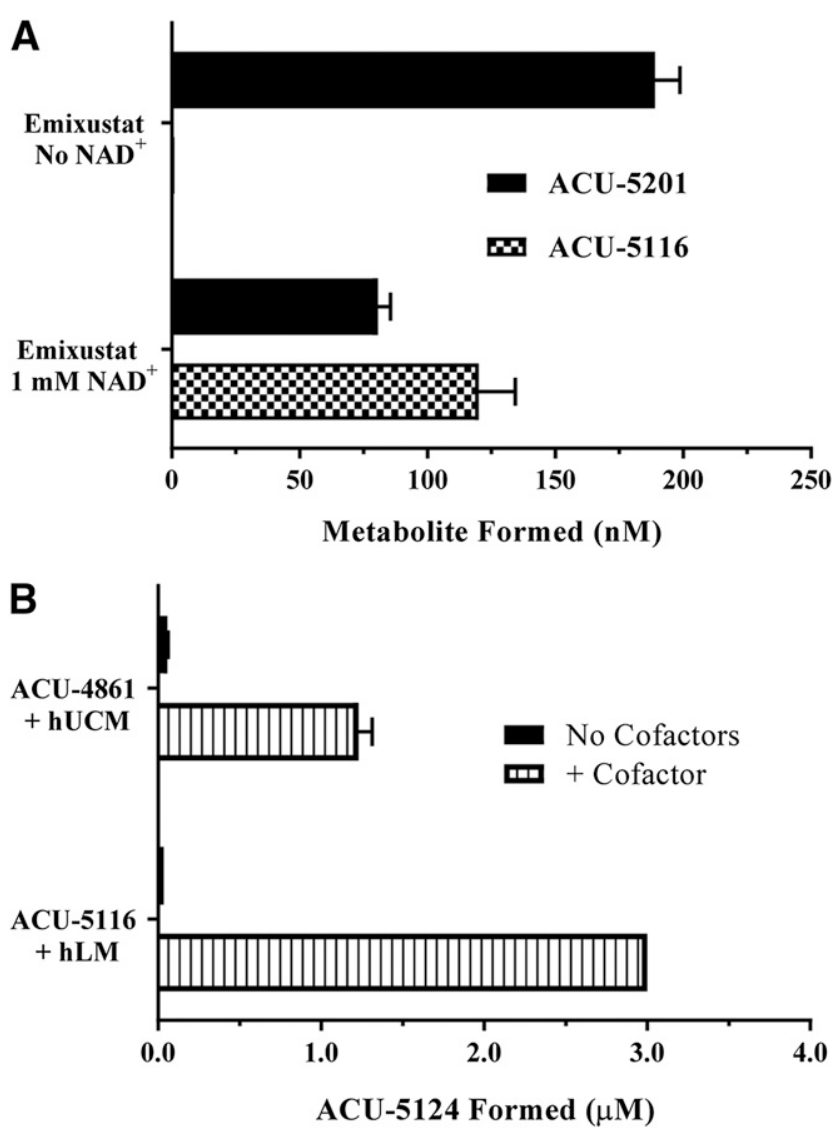

Fig. 2. Sequential metabolism of emixustat in vitro. (A) Formation of the primary deaminated metabolite ACU-5116 in hUCM with and without NAD ${ }^{+}$. Solid black bars represent amount of ACU-5201 (aldehyde), and checkered bars are amount of ACU-5116 (carboxylic acid). (B) Formation of the secondary deaminated cyclohexanol metabolite ACU-5124 from ACU-4861 (cyclohexanol hydroxypropylamine) in hUCM with $\mathrm{NAD}^{+}$(top) and from ACU-5116 in hLM with NADPH (bottom). Solid black bars represent amount without cofactors, and hatched bars represent amount formed with cofactors.

assessment of emixustat as a potential victim of in vitro drug interactions driven by inhibition of VAP-1.

Enzyme kinetics were assessed for emixustat deamination activity with the linearly optimized protein concentration (or volume of plasma) and incubation time for rhVAP-1, hUCM, hAM, and hP. MichaelisMenten kinetic constants were determined by fitting the rate equation for

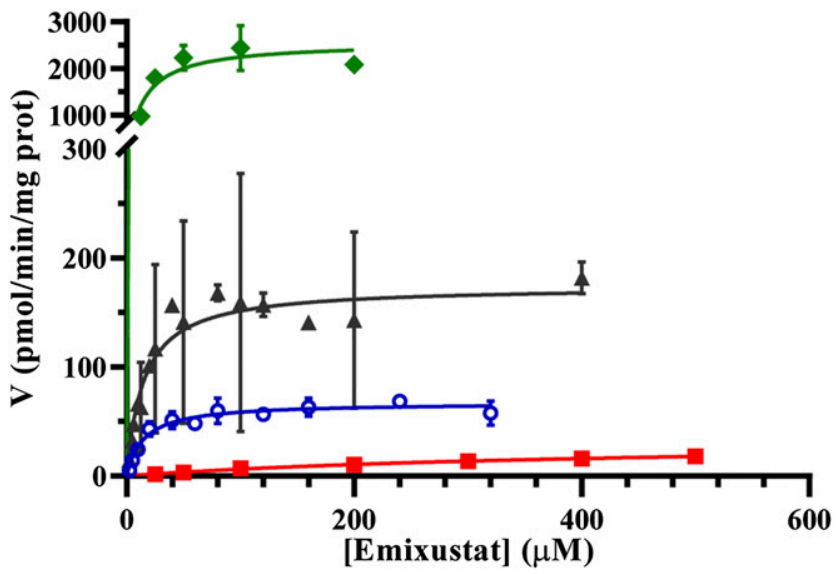

Fig. 3. Substrate saturation curves of emixustat oxidative deamination in rhVAP-1 (green diamonds), hUCM (black triangles), hAM (blue circles), and hP (red squares). a single enzyme using nonlinear regression; substrate saturation data are presented graphically in Fig. 3. Mean VAP-1 enzyme kinetic constants in rhVAP-1, and pooled hUCM, hAM, and hP are presented in Table 1 . The apparent Michaelis constant $\left(\mathrm{K}_{\mathrm{M}}\right)$ values of $13,14.7$, and $15.6 \mu \mathrm{M}$ in rhVAP-1, hUCM, and hAM, respectively, for the formation of ACU-5201 are in very close agreement, indicating that the different human sources of the VAP-1 enzyme had the same binding affinity for emixustat. These data confirmed that in vitro product formation of the aldehyde metabolite was adequate to assess the oxidative deamination of emixustat. Limited turnover of emixustat was observed in $\mathrm{hP}$. The reaction rates did not reach saturating conditions, resulting in an overestimation of the apparent $\mathrm{K}_{\mathrm{M}}$ in $\mathrm{hP}$ when compared with other VAP-1 sources.

\section{In Vitro VAP-1 Oxidative Deamination Activity}

To correlate the amine oxidase activity in human vascular membrane preparations (hUCM and hAM) and plasma with emixustat oxidative deamination rates, we adapted an assay for the commonly used amine oxidase substrate, BA, which yields BAL. Although BA is not a physiologic substrate for VAP-1, there are numerous assays based on the oxidative deamination of $\mathrm{BA}$ to form $\mathrm{BAL}$ as a marker substrate for quantifying various amine oxidase activities, including radiometric detection of extracted ${ }^{14} \mathrm{C}-\mathrm{BAL}$ (Lizcano et al., 1998), HPLC with fluorometric detection of derivatized BAL (van Dijk et al., 1995), UV monitoring of BAL (Heuts et al., 2011), and measurement of the $\mathrm{H}_{2} \mathrm{O}_{2}$ produced using Amplex red (Holt et al., 1997). A common method used for the measurement of aldehydes is an acid-catalyzed derivatization with DNPH followed by HPLC with UV or fluorometric detection (Claud et al., 2002). A direct-injection LC-MS/MS method of the DNPH-derivatized BAL (BALDNP) was developed to measure the amount of BAL formed per minute per milligram of protein in in vitro incubations. Following the initial experiments in hUCM, the LC-MS/ MS method for BALDNP was modified slightly to improve the sensitivity of the assay to better characterize inhibition in latter experiments with hAM and hP. Efficiency and recovery of the DNPH derivatization were improved by increasing the $\mathrm{pH}$ of the reaction stop solvent closer to 4.5 with $0.1 \mathrm{M}$ ammonium acetate added to the organic solvent. These changes improved the recovery BALDNP from $\sim 30 \%-$ $40 \%$ across the calibration range to $>85 \%$ when compared with neat BALDNP-spiked samples (data on file).

Enzyme kinetics were assessed for BA deamination activity with the linearly optimized protein concentration and incubation time for hUCM, $\mathrm{hAM}$, and hP (plasma volume). The Michaelis-Menten kinetic constants were determined by fitting the rate equation for a single enzyme using nonlinear regression. Mean VAP-1 enzyme kinetic constants in rhVAP1 , and pooled hUCM, hAM, and hP are presented in Table 2. The apparent BA $\mathrm{K}_{\mathrm{M}}$ values for the formation of BAL were 56.6, 78.7, 78.9, and $75.2 \mu \mathrm{M}$ in rhVAP-1 and pooled hUCM, hAM, and hP, respectively. These $\mathrm{K}_{\mathrm{M}}$ values are similar to the $63.5 \pm 9.5 \mu \mathrm{M}$

TABLE 1

Michaelis-Menten kinetic parameters for the oxidative deamination of emixustat in human sources of VAP-1

\begin{tabular}{lcc}
\hline Test System & $\mathrm{K}_{\mathrm{M}}$ & $\mathrm{V}_{\max }$ \\
\hline & $\mu M$ & pmol/min/mg protein \\
rhVAP-1 $_{\mathrm{hUCM}^{a}}$ & 13.3 & 2550 \\
$\mathrm{hAM}^{a}$ & $14.7 \pm 1.70$ & $309 \pm 176$ \\
$\mathrm{hP}$ & $15.6 \pm 7.18$ & $67.6 \pm 7.98$ \\
\hline
\end{tabular}

${ }^{a}$ Mean \pm S.D. of $n=3$ different occasions, each in triplicate. 
TABLE 2

Michaelis-Menten kinetic parameters for the oxidative deamination of benzylamine in human sources of VAP-1

\begin{tabular}{lcc}
\hline Test System & $\mathrm{K}_{\mathrm{M}}$ & $\mathrm{V}_{\max }$ \\
\hline & $\mu M$ & nmol/min/mg protein \\
$\mathrm{rhVAP}^{a}$ & $56.6 \pm 32.7$ & $1040 \pm 506$ \\
$\mathrm{hUCM}^{a}$ & 80.0 & 3.09 \\
$\mathrm{hAM}^{a}$ & $78.9 \pm 33.8$ & $65.6 \pm 4.64$ \\
$\mathrm{hP}^{b}$ & $75.2 \pm 4.56$ & $0.467 \pm 0.024(17.6 \mathrm{nmol} / \mathrm{ml} / \mathrm{h})$ \\
\hline
\end{tabular}

${ }^{a}$ Mean \pm S.D. of $n=4$ different occasions, each in triplicate.

${ }^{b}$ Mean \pm S.D. of $n=3$ different occasions, two in duplicate and one in triplicate.

previously reported for BA deamination in human umbilical artery microsomes (Claud et al., 2002), indicating that the different human sources of the VAP-1 enzyme had the same binding affinity for this marker substrate. Although the rates of product formation in plasma were much lower than in membranes or recombinant sources, the $\mathrm{V}_{\max }$ in pooled hP was $17.6 \mathrm{nmol} / \mathrm{ml} / \mathrm{h}$, which is comparable to other reported clinical values for diabetic versus normal subjects, with means of 20.6 and $14.3 \mathrm{nmol} / \mathrm{ml} / \mathrm{h}$, respectively (Garpenstrand et al., 1999), and chronic liver disease versus healthy normal subjects, with means of 18.8 and $10.7 \mathrm{nmol} / \mathrm{ml} / \mathrm{h}$, respectively (Kurkijärvi et al., 2000).

\section{Reaction Phenotyping of VAP-1 Oxidative Deamination}

Correlation Analysis. The interindividual variability in VAP-1 activity observed across a population of tissue donors was assessed in hUCM, hAM, and hP acquired from commercial sources. Saturating concentrations of emixustat and BA were incubated in triplicate for the individual sources of VAP-1 at the linearly optimized conditions. Mean rates of ACU-5201 formation ranged from 36.4 to $170,5.82$ to 100 , and 0.748 to $7.51 \mathrm{pmol} / \mathrm{min} / \mathrm{mg}$ protein in hUCM, hAM, and hP, respectively. The 10-fold difference in emixustat deamination in $\mathrm{hP}$ and 17-fold difference in hAM had more variability than the 4.7-fold difference in hUCM; however, the mean rate of 20 individuals in both hUCM and hAM was very similar at 44.7 and $49.4 \mathrm{pmol} / \mathrm{min} / \mathrm{mg}$ protein, respectively. Mean rates of BAL product formation ranged from 13.7 to $40.4,4.16$ to 36.6 , and 0.0637 to $1.17 \mathrm{nmol} / \mathrm{min} / \mathrm{mg}$ protein in hUCM, hAM, and hP, respectively. The 18-fold difference in BA deamination in $\mathrm{hP}$ and 9-fold difference in hAM also exhibited more variability than the 3-fold difference in hUCM. The interindividual variability in VAP-1 protein levels measured by ELISA was also assessed across a population of tissue donors in hUCM, hAM, and hP. Mean levels of VAP-1 protein measured ranged from 267 to 1690 ,
168 to 506 , and 0.329 to $1.10 \mathrm{ng} / \mathrm{mg}$ protein in hUCM, hAM, and hP, respectively. The variability of VAP-1 measured by ELISA normalized to total protein varied less (3- to 6-fold) than the oxidative deamination activities.

Correlation constants for comparisons of individual BA deamination rates and VAP-1 protein levels to each other and to emixustat deamination activity using both Gaussian (Pearson) and nonparametric (Spearman) analyses are presented in Table 3. Correlations of individual BA deamination rates and VAP-1 protein levels to emixustat deamination activity are graphically presented in Fig. 4. VAP-1 BA deamination activity was highly correlated with the VAP-1 levels in plasma and significantly correlated in hUCM and hAM, supporting the use of BA deamination as a marker for VAP-1 activity. Benzylamine deamination activity and VAP-1 protein levels were well correlated with emixustat deamination in hUCM, hAM, and hP. These results indicate that interindividual variability in VAP-1 contributes to variability of emixustat deamination. Also of interest, the plasma activity or protein levels were not predictive of the oxidative deamination activity in the elderly human aorta membranes.

Recombinant Human Enzymes. Recombinant forms of human VAP-1, LOXL2 (another amine oxidase containing copper), MAO-A, and MAO-B (BD Gentest, Franklin Lakes, NJ) were also used to assess the specificity of this amine oxidase activity in deaminating emixustat to ACU-5201. As noted earlier, rhVAP-1 had an affinity to emixustat similar to the other human tissue sources of VAP-1 with high rates of maximum reaction velocity (Fig. 3; Table 1), affirming the deaminase activity of this enzyme for emixustat. No apparent metabolites of radiolabeled emixustat were evident after incubation with recombinant human MAO-A (rhMAO-A) and rhMAO-B for 30 minutes, whereas activities for the positive control substrates kynuramine and benzylamine confirmed active monoamine oxidases (data on file). In addition, no ACU-5201 was observed when incubating emixustat with recombinant human LOXL2 for 30 minutes.

Selective Chemical Inhibitors. Selective inhibition of amine oxidase activity was also used to assess the contribution of VAP-1 and MAO-A and $-\mathrm{B}$ in both hUCM and hAM. Semicarbazide and LJP-1207 were coincubated with emixustat as specific inhibitors of VAP-1, and clorgyline and deprenyl were coincubated to selectively inhibit MAO-A and MAO-B, respectively. Quinidine was tested because it is commonly used in clinical drug-interaction studies as a CYP2D6 inhibitor [Table 12 Food and Drug Administration Drug Development and Drug Interactions: Table of Substrates, Inhibitors and Inducers] and its potential effects on amine oxidase activity have not been established. Figure 5 shows the effects of high and low concentrations (10-fold difference) of each inhibitor to assess dose dependence; however, the

TABLE 3

Correlations of in vitro emixustat oxidative deamination metabolism, benzylamine deamination, and VAP-1 levels

\begin{tabular}{lccccc}
\hline \multicolumn{1}{c}{ Correlation } & \multicolumn{2}{c}{ Spearman } & & \multicolumn{2}{c}{ Pearson } \\
\cline { 2 - 3 } \cline { 5 - 6 } & $r$ & Two-Tailed $P$ & & $r$ & Two-Tailed $P$ \\
\hline hUCM emixustat deamination vs. hUCM BA deamination & 0.705 & $<0.001$ & & 0.736 & 0.0018 \\
hUCM emixustat deamination vs. hUCM VAP-1 levels & 0.827 & $<0.001$ & & 0.886 & $<0.0001$ \\
hUCM BA deamination vs. hUCM VAP-1 levels & 0.639 & $<0.005$ & & 0.627 & $<0.005$ \\
hAM emixustat deamination vs. hAM BA deamination & 0.794 & $<0.00001$ & & 0.823 & $<0.00001$ \\
hAM emixustat deamination vs. hAM VAP-1 levels & 0.789 & $<0.00001$ & & 0.732 & 0.0002 \\
hAM BA deamination vs. hAM VAP-1 levels & 0.543 & 0.013 & & 0.568 & 0.009 \\
hP emixustat deamination vs. hP BA deamination & 0.922 & $<0.00001$ & & 0.821 & 0.0002 \\
hP emixustat deamination vs. hP VAP-1 levels & 0.874 & $<0.00001$ & & 0.764 & 0.0009 \\
hP BA deamination vs. hP VAP-1 levels & 0.911 & $<0.00001$ & & 0.913 & $<0.00001$ \\
hAM emixustat deamination vs. hP emixustat deamination & 0.582 & 0.025 & & 0.629 & 0.012 \\
hAM emixustat deamination vs. hP BA deamination & 0.444 & 0.069 & & 0.531 & 0.023 \\
hAM emixustat deamination vs. hP VAP-1 & 0.486 & 0.041 & & 0.477 & 0.045
\end{tabular}



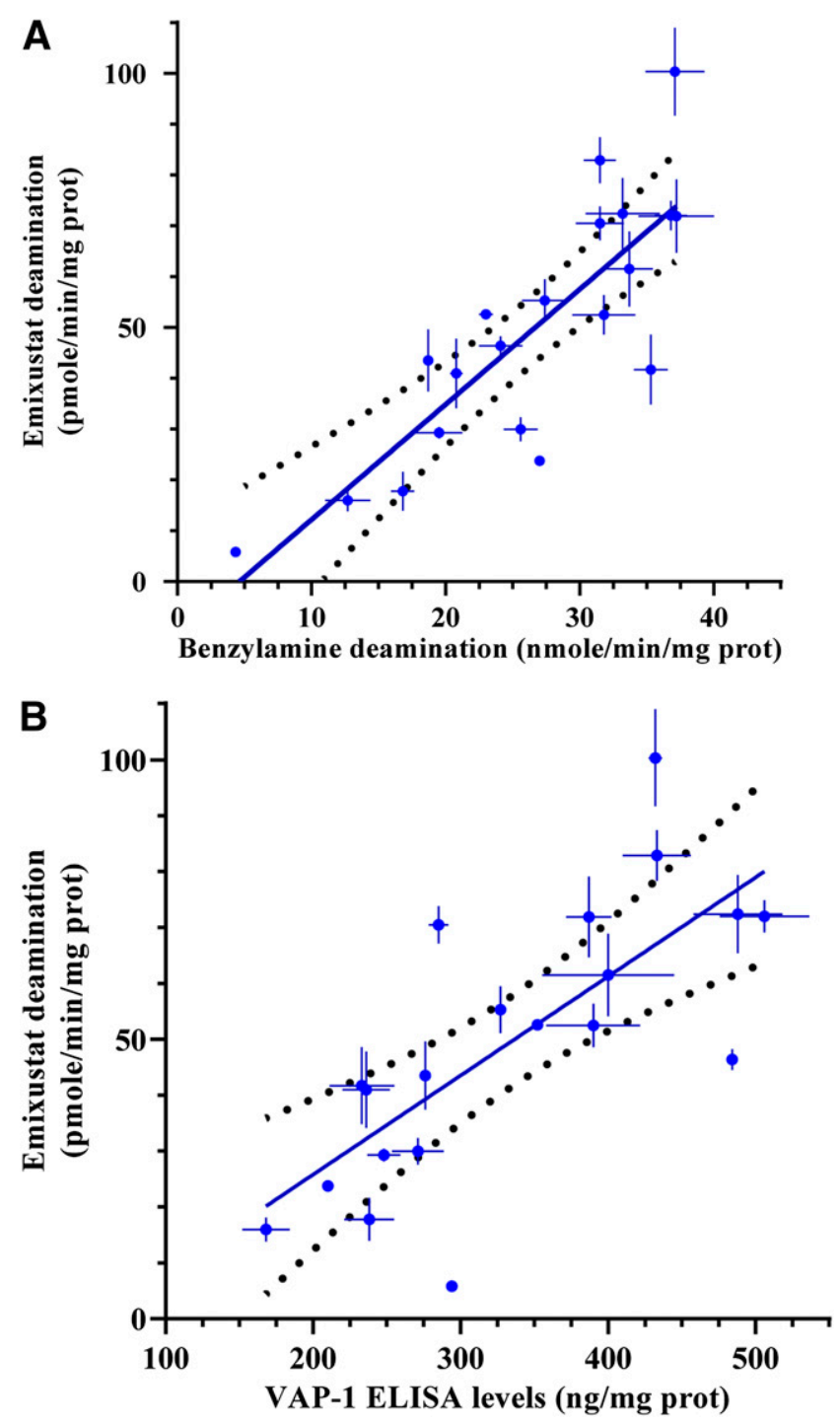

Fig. 4. Correlation of emixustat oxidative deamination with VAP-1 marker activity (A) and protein levels (B) in 20 individually derived sources of hAM. Blue circles with bidirectional error bars ( $n=3$ for activity and $n=2$ for ELISA) represent individual hAM values, solid blue line represents linear regression, and black dots represent $95 \%$ confidence interval.

high and low concentrations were not the same for the different compounds since low concentrations were chosen based on available maximum plasma concentrations or in vitro $\mathrm{IC}_{50}$ values. LJP-1207 had the most pronounced effect on emixustat deamination with complete inhibition at $1.0 \mu \mathrm{M}$. Semicarbazide had moderate effects at $10 \mu \mathrm{M}$ but more complete inhibition at $100 \mu \mathrm{M}$. Clorgyline and deprenyl did not inhibit emixustat deamination in hUCM at the low concentration of $50 \mu \mathrm{M}$, but both inhibited at $500 \mu \mathrm{M}$. Neither clorgyline nor deprenyl inhibited at 10 and $100 \mu \mathrm{M}$ in hAM. Quinidine did not inhibit oxidative deamination of emixustat in vitro, nor did $\beta$-aminoproprionitrile (a LOX-specific inhibitor; Mercier et al., 2009) in hAM, even at concentrations as high as $500 \mu \mathrm{M}$.

\section{In Vitro Drug-Drug Interaction Potential}

Emixustat as the Victim. The reaction phenotyping for oxidative deamination of emixustat by VAP-1 infers its predominant role in the biotransformation to the three major metabolites observed in human plasma. Since metabolic clearance by VAP-1 is an uncommon pathway,
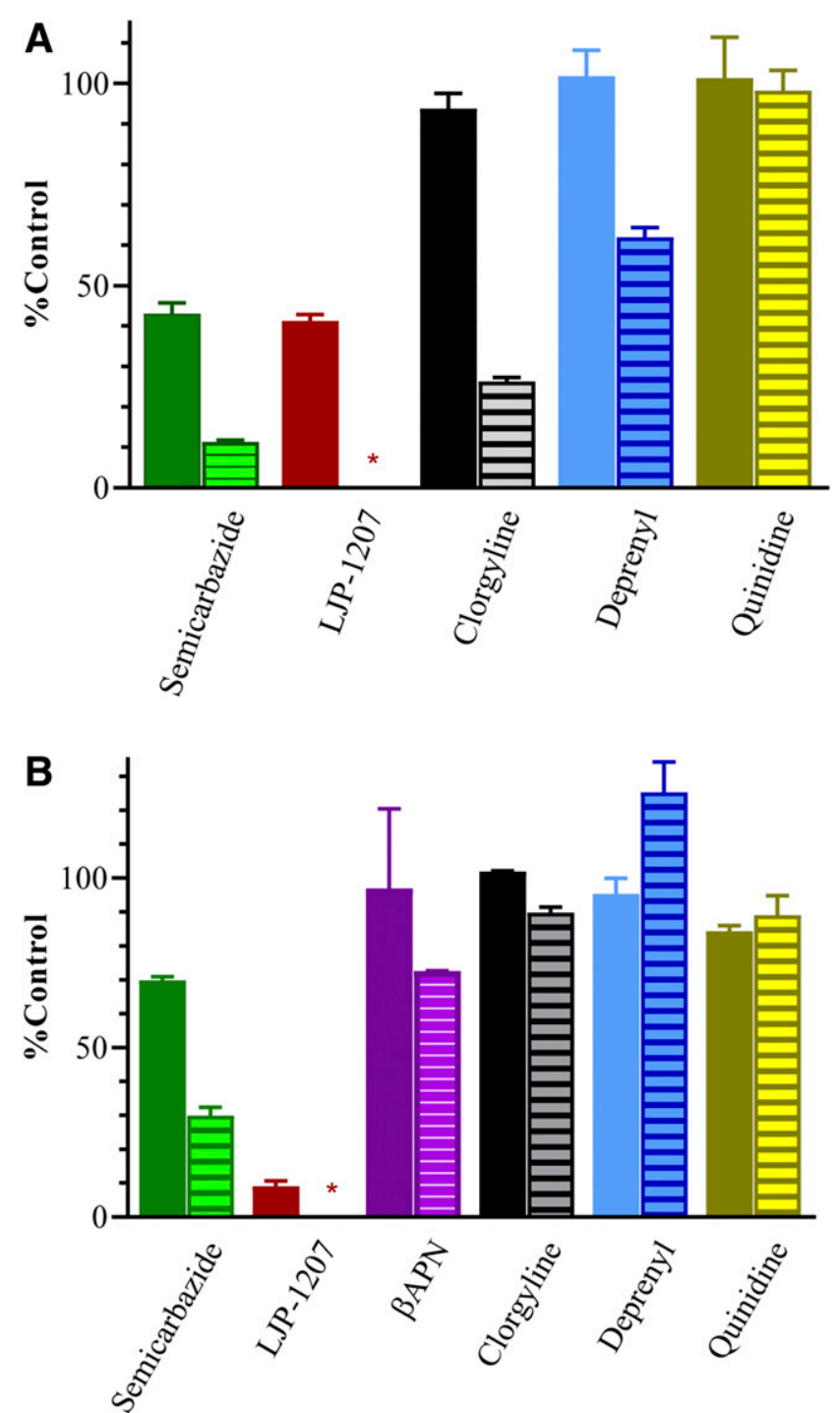

Fig. 5. Selective chemical inhibition of emixustat oxidative deamination in pooled hUCM (A) and pooled hAM (B). Solid and striped bars represent low and high inhibitor concentrations, respectively, with error bars showing S.D. of triplicate assays. Semicarbazide (green) concentrations were 10 and $100 \mu \mathrm{M}$ in both hUCM and hAM, LJP-1207 (maroon) concentrations were 0.1 and $1.0 \mu \mathrm{M}$ in both hUCM and hAM, $\beta$-aminoproprionitrile ( $\beta \mathrm{APN}$; LOX inhibitor; purple) concentrations were 50 and $500 \mu \mathrm{M}$ in hAM only (B), clorgyline (black; MAO-A inhibitor) and deprenyl (blue; MAO-B inhibitor) concentrations were 50 and $500 \mu \mathrm{M}$ in hUCM and 10 and $100 \mu \mathrm{M}$ in hAM, and quinidine (olive) concentrations were 1 and $10 \mu \mathrm{M}$ in both hUCM and hAM.

its sensitivity to inhibition by other drugs as an underlying mechanism for DDIs has been seldomly scrutinized. To assess the potential for emixustat exposure to increase in response to being victimized by an inhibitor of VAP-1, in vitro screening for VAP-1 inhibition was conducted following review of therapeutic agents with various functional moieties (e.g., hydrazine, guanidine, phthalazine) that have been demonstrated to inhibit VAP-1 activity. Additionally, inhibitors of MAO isoforms were included because they often have overlapping inhibition profiles with VAP-1/SSAO (Kinemuchi et al., 2004; Dunkel et al., 2008). Various potential inhibitors (15 compounds in total) were screened to assess their potential to inhibit the formation of ACU5201 in pooled hAM at a $60-\mu \mathrm{M}$ concentration of emixustat, which was less than maximal saturation but high enough to remain sensitive to inhibition in pooled hAM, pooled hP, and rhVAP-1. Inhibition of 
benzylamine deamination was similarly screened at $80 \mu \mathrm{M}$. Most compounds were screened at a low $(2$ or $10 \mu \mathrm{M})$ and high $(20$ or $100 \mu \mathrm{M})$ concentration. Low concentrations were chosen based on available maximum plasma concentrations or published in vitro $\mathrm{IC}_{50}$ values for their targets, and high concentrations were 10 times the lower concentration. If a compound exhibited $>50 \%$ inhibition at either of the screening concentrations, an $\mathrm{IC}_{50}$ was subsequently determined. Results are presented in Supplemental Table 1.

Guanabenz $(20 \mu \mathrm{M})$, hydralazine $(20 \mu \mathrm{M})$, and semicarbazide $(100 \mu \mathrm{M})$ inhibited the formation of ACU-5201 by $69 \%, 87 \%$, and $70 \%$, respectively. The specific VAP-1 inhibitor, LJP-1207, also inhibited oxidative deamination of emixustat by more than $50 \%$; its $\mathrm{IC}_{50}$ was $75 \mathrm{nM}$, as determined in earlier hUCM experiments. When tested for inhibition of emixustat deamination in pooled hAM, $\mathrm{IC}_{50}$ values for guanabenz, hydralazine, and isoniazid were 10.2, 7.4, and $55.4 \mu \mathrm{M}$, respectively. $\mathrm{IC}_{50}$ values for guanabenz and hydralazine were 46.9 and $6.4 \mu \mathrm{M}$ when tested for inhibition of BA deamination in pooled hAM, and 9.46 and $26.5 \mu \mathrm{M}$ in rhVAP-1, respectively. Anticipated results were observed as reported earlier for MAO-specific (clorgyline and deprenyl) and LOX-specific ( $\beta$-aminoproprionitrile) inhibitors. The CYP2D6 inhibitors paroxetine and quinidine also did not inhibit. Although benserazide (Boomsma et al., 1995) and caffeine (Olivieri and Tipton, 2011) are reported to be inhibitors of plasma VAP-1, and antidepressants have been observed to inhibit BA deamination, these compounds were not potent in vitro inhibitors of emixustat or BA deamination in vascular membranes.

Emixustat as a Perpetrator. Inhibitory potential of emixustat as a perpetrator against VAP-1-mediated BA $(80 \mu \mathrm{M})$ deamination activity in hUCM and hAM yielded $\mathrm{IC}_{50}$ values of 161 and $109 \mu \mathrm{M}$, respectively. During in vitro incubations of human plasma, $100 \mu \mathrm{M}$ emixustat inhibited BA deamination by $<30 \%$. More thorough kinetic analyses to determine the $\mathrm{K}_{\mathrm{i}}$ value were performed in hUCM with emixustat concentrations of 100, 200, and $400 \mu \mathrm{M}$ as shown in Fig. 6, yielding a $\mathrm{K}_{\mathrm{i}}$ value of $186 \mu \mathrm{M}$ using a mixed inhibition model.

Since VAP-1 appears to be upregulated in several human pathologies (Pannecoeck et al., 2015), there are numerous published reports of VAP1 inhibition as a potential anti-inflammatory therapy (Kinemuchi et al., 2004; Salter-Cid et al., 2005; Dunkel et al., 2008). This study did not establish an in vitro system to examine the potential to chemically induce VAP-1, but observations were made regarding VAP-1 variability across the donor tissues. Greater variability in VAP-1 activity in the elderly aorta tissue compared with umbilical cords suggests that emixustat clearance may be more variable in an elderly population. Mean rates of emixustat deamination via vascular tissue (membrane-bound) and soluble (plasma) VAP-1 in elderly individuals were evaluated using two-way analyses of variance to determine if in vitro emixustat VAP-1 metabolism differences were discernable between select subpopulations of the demographic data in this small population of donors. There was no statistical significance between males and females, nor between patients with diabetes and nonpatients with diabetes. However, Caucasians did have significantly higher emixustat metabolic rates than non-Caucasians in hAM $(P<0.005$, membrane bound), but not in hP (soluble).

\section{Clinical Pharmacokinetics}

The mean plasma concentration-time profiles and noncompartmental PK parameters of emixustat and its three major metabolites (ACU-5116, ACU-5124, and ACU-5149) following daily emixustat doses of 2.5, 5, and $10 \mathrm{mg} / \mathrm{kg}$ for 7 days are shown in Fig. 7 and Tables 4 and 5 , respectively. Mean plasma concentrations at each sample collection time point for all three metabolites were generally 10-fold higher than
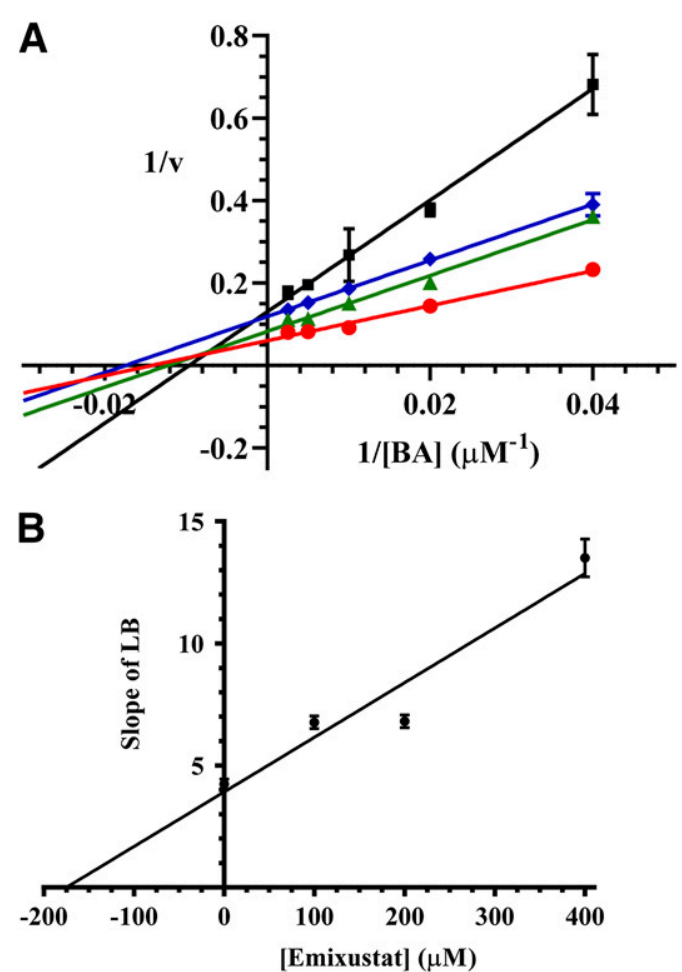

Fig. 6. Inhibition kinetics of emixustat on benzylamine deamination in hUCM. (A) Lineweaver-Burke (LB) plot with inverse BA concentrations and inverse activity rates as the $x$ - and $y$-axes, respectively. Red circles, green triangles, blue diamonds, and black squares represent coincubated emixustat concentrations of $0,100,200$, and $400 \mu \mathrm{M}$, respectively. (B) Plot of the LB slopes versus emixustat concentrations to determine $\mathrm{K}_{\mathrm{i}}$.

emixustat, and the metabolite plasma concentration profiles declined in parallel with emixustat on both days 1 and 7 (Fig. 7). Maximum plasma concentrations $\left(\mathrm{C}_{\max }\right)$ of emixustat $(\sim 11 \mathrm{nM}$ at $10 \mathrm{mg}$ once daily $)$ and its three deaminated metabolites increased dose proportionally over the dose range, and the average time to reach $\mathrm{C}_{\max }\left(\mathrm{T}_{\max }\right)$ ranged between 2.1 and 4.1 hours for all four analytes at all three dose levels, indicating rapid metabolism, with ACU-5116 having the earliest peak concentrations. Comparable to $\mathrm{C}_{\max }$ values, AUC values for emixustat and its three metabolites ranged approximately 4- to 6-fold across the 18 individuals in each cohort, within each dose level, and their mean values increased in an apparent dose-proportional manner on both days 1 and 7, consistent with linear kinetics over the 2.5- to 10-mg dose range. AUC-based metabolite-to-parent ratios indicated plasma exposures to each inactive metabolite were 6- to 11-fold greater than to the pharmacologically active parent. Accumulation ratios of exposure (based on AUC) on day 7 ranged from 1.09 to 1.31 for emixustat and its three metabolites, indicating minimal accumulation for any of the analytes as predicted since their elimination half-lives $\left(\mathrm{t}_{1 / 2}\right)$ were a fraction of the dosing interval (24 hours). Mean elimination half-lives of emixustat and its three metabolites were between 5.9 and 7.1 hours.

\section{Discussion}

In a previous human ADME study, three major deaminated metabolites of emixustat were identified in the plasma following a single 40-mg dose: ACU-5116, ACU-5124, and ACU-5149 (Fitzsimmons et al., 2018). The clinical PK results in subjects with geographic atrophy presented here confirmed that these three deaminated metabolites predominate in plasma following single and multiple daily oral doses 

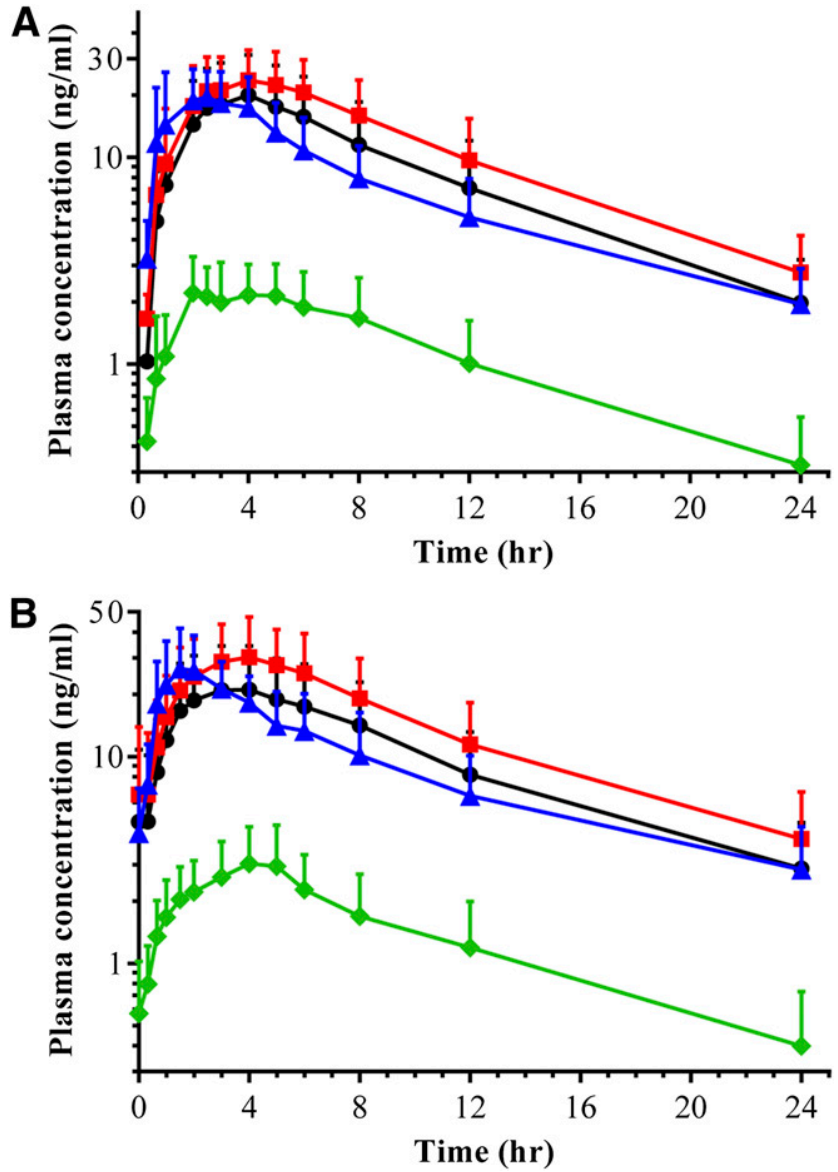

Fig. 7. Clinical pharmacokinetics of emixustat and its three major deaminated metabolites following a single $10-\mathrm{mg} / \mathrm{kg}$ oral dose (A) and following a daily $10 \mathrm{mg} / \mathrm{kg}$ dose (B) on day 7 in 18 individuals with geographic atrophy. Plasma concentrations of emixustat (green diamonds), ACU-5116 (blue triangles), ACU5124 (red squares), and ACU-5149 (black circles).

and reach maximum concentrations rapidly, demonstrating formationrate-limited elimination with a similar $\mathrm{t}_{1 / 2}$ to the parent (Fig. 7; Table 2).

Inconsistent with these in vivo results, in vitro metabolism of emixustat in human hepatocytes resulted in a large fraction of emixustat
( $\sim 34 \%$ ) being unchanged, with the oxidatively deaminated metabolites each accounting for a smaller fraction $(4 \%-9 \%)$ of the ${ }^{14} \mathrm{C}$ after 2 hours. The remaining metabolites observed in human hepatocytes were mainly cyclohexanol metabolites and a cyclohexanone metabolite of the hydroxypropylamine, yet none of these metabolites were $\geq 10 \%$ of the circulating plasma radioactivity. Although the secondary hydroxylated metabolites, ACU-5124 and ACU-5149, both achieved $>10 \%$ of the total circulating plasma radioactivity, the primary metabolite, ACU5116, appeared in human plasma more quickly than both of the major secondary metabolites, emixustat (see Fig. 7), or any of the other minor metabolites (Fitzsimmons et al., 2018). This suggests that formation of the major secondary metabolites occurs primarily via monohydroxylation of ACU-5116 by P450 isoform(s) (Figs. 1 and 2).

Although in vivo formation of carboxylic acid metabolites from a primary amine is not a common xenobiotic biotransformation, one example is primaquine (PQ), whose major human plasma metabolite is the carboxylic acid carboxyprimaquine (CPQ). Plasma levels of CPQ rapidly exceeded PQ concentrations, with the $\mathrm{C}_{\max }$ of CPQ 10-fold higher than the parent. Even though $64 \%$ of the radioactivity of a single ${ }^{14} \mathrm{C}-\mathrm{PQ}$ oral dose was recovered in the urine, less than $4 \%$ was due to PQ (Mihaly et al., 1984). In vivo biotransformation of PQ to a carboxylic acid had similar conversion kinetics to that observed for emixustat and its primary carboxylic acid metabolite, ACU-5116. The high rates of emixustat deamination motivated an early study that collected portal vein blood from rats. High levels of ACU-5116, ACU-5124, and ACU5149 were observed presystemically within 15 minutes of an emixustat oral dose (data on file).

In vitro investigations into the sequential metabolism of PQ determined that initial conversion of PQ to an aldehyde via MAO-A (Constantino et al., 1999; Pybus et al., 2012) was followed by further oxidation to CPQ by aldehyde dehydrogenase (Frischer et al., 1991). This in vitro sequential metabolism of $\mathrm{PQ}$ is similar to the conversion of emixustat to ACU-5116, where the initial aldol product of VAP-1 (see Fig. 1) is converted to the carboxylic acid only when $\mathrm{NAD}^{+}$is added (Fig. 2A). A similar pattern was described for tresperimus wherein aldehyde metabolites were predominantly observed following its incubation in plasma in vitro, while acid metabolites were also observed in plasma collected from humans and rats dosed with tresperimus (Claud et al., 2001). Because high levels of emixustat's acid metabolites were observed in the portal circulation of rats (as noted earlier), gastric mucosal and small intestinal mucosal homogenate human tissue

TABLE 4

Mean pharmacokinetic parameters on day 1 for emixustat and its major metabolites (ACU-5116, ACU-5124, and ACU-5149) in subjects with geographic atrophy

\begin{tabular}{|c|c|c|c|c|c|}
\hline Analyte & $\mathrm{C}_{\max } \pm$ S.D. $(\mathrm{N})$ & $\mathrm{T}_{\max } \pm$ S.D. $(\mathrm{N})$ & $\mathrm{t}_{1 / 2} \pm$ S.D. (N) & $\mathrm{AUC}_{0-24} \pm$ S.D. $(\mathrm{N})$ & $\mathrm{AUC}_{0-\infty} \pm$ S.D. (N) \\
\hline & $n g / m l$ & $h$ & $h$ & $n g \cdot h / m l$ & $n g \cdot h / m l$ \\
\hline \multicolumn{6}{|c|}{$2.5 \mathrm{mg}$ QD emixustat } \\
\hline Emixustat & $0.637 \pm 0.279$ & $4.31 \pm 1.83(17)$ & $5.84 \pm 1.83(16)$ & $6.42 \pm 3.39(15)$ & $7.15 \pm 3.82(12)$ \\
\hline ACU-5116 & $5.96 \pm 2.28(17)$ & $2.59 \pm 0.895(17)$ & $5.38 \pm 1.96(17)$ & $46.1 \pm 16.1(10)$ & $50.0 \pm 21.8(8)$ \\
\hline ACU-5124 & $7.29 \pm 3.89(17)$ & $4.14 \pm 0.956(17)$ & $5.72 \pm 1.84(16)$ & $76.8 \pm 42.2(9)$ & $80.3 \pm 52.7(8)$ \\
\hline ACU-5149 & $5.68 \pm 3.02(17)$ & $3.72 \pm 0.966(17)$ & $5.44 \pm 1.76(16)$ & $53.1 \pm 41.6(9)$ & $59.0 \pm 49.3(8)$ \\
\hline \multicolumn{6}{|c|}{$5.0 \mathrm{mg}$ QD emixustat } \\
\hline Emixustat & $1.61 \pm 0.832(18)$ & $3.47 \pm 1.91(18)$ & $6.35 \pm 1.05(17)$ & $14.0 \pm 6.15(18)$ & $15.0 \pm 6.96(17)$ \\
\hline ACU-5116 & $12.0 \pm 5.91(18)$ & $2.24 \pm 1.35(18)$ & $5.78 \pm 2.66(18)$ & $81.4 \pm 34.9(17)$ & $94.2 \pm 44.7(17)$ \\
\hline ACU-5124 & $13.6 \pm 6.79(18)$ & $3.61 \pm 1.10(18)$ & $5.57 \pm 1.08(18)$ & $129 \pm 72.8$ & $143 \pm 79.6(17)$ \\
\hline ACU-5149 & $11.0 \pm 6.18(18)$ & $3.42 \pm 1.27(18)$ & $5.41 \pm 1.72(18)$ & $101 \pm 70.9$ & $110 \pm 79.9(17)$ \\
\hline \multicolumn{6}{|c|}{$10.0 \mathrm{mg}$ QD emixustat } \\
\hline Emixustat & $2.79 \pm 1.18(18)$ & $3.14 \pm 1.49(18)$ & $6.78 \pm 1.40(18)$ & $27.4 \pm 12.5(18)$ & $30.8 \pm 15.3(18)$ \\
\hline ACU-5116 & $23.4 \pm 9.04(18)$ & $2.66 \pm 1.08$ & $6.60 \pm 2.18$ & $168 \pm 61.7(18)$ & $188 \pm 70.6(16)$ \\
\hline ACU-5124 & $25.3 \pm 9.84(18)$ & $4.04 \pm 1.14(18)$ & $6.29 \pm 1.36$ & $263 \pm 122(18)$ & $288 \pm 133(18)$ \\
\hline ACU-5149 & $21.1 \pm 10.9$ & $3.87 \pm 1.25(18)$ & $6.12 \pm 1.42(18)$ & $204 \pm 117(18)$ & $223 \pm 128$ \\
\hline
\end{tabular}

$\mathrm{AUC}_{0-24}$, area under the plasma concentration-time curve from time 0 to 24 hours; $\mathrm{AUC}_{0-\infty}$ area under the plasma concentration-time curve from time 0 to infinity; $\mathrm{C}_{\max }$, maximum observed plasma concentration; $\mathrm{N}$, number of subjects; $\mathrm{QD}$, once daily; $\mathrm{t}_{1 / 2}$, terminal half-life; $\mathrm{T}_{\max }$, time to reach $\mathrm{C}_{\max }$. 
TABLE 5

Mean pharmacokinetic parameters on day 7 for emixustat and its major metabolites (ACU-5116, ACU-5124, and ACU-5149) in subjects with geographic atrophy

\begin{tabular}{lccccc}
\hline \multicolumn{1}{c}{ Analyte } & $\mathrm{C}_{\max } \pm$ S.D. $(\mathrm{N})$ & $\mathrm{T}_{\max } \pm$ S.D. $(\mathrm{N})$ & $\mathrm{t}_{1 / 2} \pm$ S.D. $(\mathrm{N})$ & AUC $_{0-t} \pm$ S.D. $(\mathrm{N})$ & $\mathrm{R}_{\mathrm{ac}} \pm$ S.D. (N) \\
\hline \multicolumn{7}{c}{$n g / m l$} & $h$ & $h$ & $n g \cdot h / m l$ & \\
2.5 mg QD emixustat & & & & & \\
Emixustat & $0.739 \pm 0.372(17)$ & $3.92 \pm 1.57(17)$ & $6.98 \pm 1.73(16)$ & $7.33 \pm 2.40(14)$ & $1.20 \pm 0.152(12)$ \\
ACU-5116 & $7.34 \pm 3.4(17)$ & $2.15 \pm 0.949(17)$ & $6.00 \pm 3.26(17)$ & $40.8 \pm 21.7(17)$ & $1.17 \pm 0.237(8)$ \\
ACU-5124 & $7.41 \pm 3.47(17)$ & $4.00 \pm 1.50(17)$ & $5.89 \pm 2.46(15)$ & $62.1 \pm 44.6(17)$ & $1.09 \pm 0.137(6)$ \\
ACU-5149 & $5.93 \pm 3.77(17)$ & $3.35 \pm 1.32(17)$ & $5.94 \pm 2.09(15)$ & $49.2 \pm 43.5(17)$ & $1.27 \pm 0.392(5)$ \\
5.0 mg QD emixustat & & & & & \\
Emixustat & $1.69 \pm 0.745(18)$ & $3.81 \pm 1.41(18)$ & $7.04 \pm 1.23(18)$ & $16.5 \pm 7.55(18)$ & $1.19 \pm 0.225(18)$ \\
ACU-5116 & $14.23 \pm 6.23(18)$ & $2.17 \pm 2.15(18)$ & $6.78 \pm 2.85(18)$ & $91.4 \pm 45.2(18)$ & $1.31 \pm 0.337(16)$ \\
ACU-5124 & $16.1 \pm 8.96(18)$ & $3.97 \pm 1.46(18)$ & $6.95 \pm 1.82(17)$ & $159 \pm 91.0(18)$ & $1.24 \pm 0.169(18)$ \\
ACU-5149 & $12.6 \pm 6.85(18)$ & $3.39 \pm 1.64(18)$ & $6.36 \pm 2.01(17)$ & $116 \pm 83.0(18)$ & $1.23 \pm 0.298(17)$ \\
10.0 mg QD emixustat & & & & & \\
Emixustat & $3.48 \pm 1.81(18)$ & $3.09 \pm 1.32(18)$ & $7.05 \pm 2.83(18)$ & $32.4 \pm 17.7(18)$ & $1.17 \pm 0.277(18)$ \\
ACU-5116 & $29.6 \pm 13.0(18)$ & $2.32 \pm 1.58(18)$ & $6.90 \pm 2.90(17)$ & $209 \pm 100(18)$ & $1.27 \pm 0.315(18)$ \\
ACU-5124 & $32.2 \pm 16.6(18)$ & $4.03 \pm 1.04(18)$ & $6.70 \pm 1.64(17)$ & $332 \pm 189(18)$ & $1.29 \pm 0.176(17)$ \\
ACU-5149 & $23.5 \pm 12.8(18)$ & $3.75 \pm 1.72(18)$ & $6.25 \pm 1.72(17)$ & $239 \pm 146(18)$ & $1.17 \pm 0.230(18)$ \\
\hline
\end{tabular}

$\mathrm{AUC}_{0-t}$, area under the plasma concentration-time curve from time 0 to the last measurable plasma concentration; $\mathrm{C}_{\mathrm{max}}$, maximum observed plasma concentration; $\mathrm{N}$, number of subjects; $\mathrm{QD}$, once daily; $\mathrm{R}_{\mathrm{ac}}$, accumulation ratio (calculated based upon $\mathrm{AUC}_{0-\infty}$ for emixustat and $\mathrm{AUC}_{0-24}$ for metabolites); $\mathrm{t}_{1 / 2}$, terminal half-life; $\mathrm{T}_{\text {max }}$, time to reach $\mathrm{C}_{\max }$

preparations were tested alongside the rhMAO enzymes. The stability of $\left[{ }^{14} \mathrm{C}\right]$ emixustat in these in vitro systems drove the decision to seek a different extrahepatic model for emixustat oxidative deamination.

The present work indicates the biotransformation of emixustat is predominantly driven by the copper-containing amine oxidase VAP-1. Vascular adhesion protein-1 was found to be identical in gene sequence and oxidative deamination function to SSAO (Smith et al., 1998), a member of the amine oxidase copper-containing gene family that is ubiquitous across nature and distinct from flavin adenine dinucleotide cofactor-containing monoamine and polyamine oxidases (Salmi and Jalkanen, 1992). As a homodimeric 180- to 200-kDa ectoenzyme with a topaquinone cofactor (Holt et al., 1998), VAP-1 is found mainly on plasma membranes of the endothelium, adipose, and smooth muscle of mammals (Wibo et al., 1980; Barrand and Callingham, 1985; Precious et al., 1988). Currently, amlodipine is the only marketed drug with oxidative deamination metabolism by a copper-containing amine oxidase (Stopher et al., 1988), yet this is a relatively minor metabolic clearance pathway. Vascular adhesion protein-1 was also established as the major metabolic clearance enzyme of the unmarketed immunosuppressive compound tresperimus (Claud et al., 2001, 2002).

Low to no yields of carboxylic acid metabolites in hepatocytes, liver microsomes, liver mitochondria, recombinant P450, and MAO enzymes were congruent with constitutive VAP-1 expression in sinusoidal endothelium within the liver (Lalor et al., 2002). The data presented here expand on the utility of endothelial membrane preparations for investigating VAP-1 oxidative deamination metabolism of xenobiotics that Claud et al. (2002) first introduced. Similar enzyme kinetics for the oxidative deamination of emixustat to ACU-5201 were observed in hUCM, hAM, and rhVAP-1 (see Table 1). The oxidative deamination activities in these sources of VAP-1 were also confirmed using BA as a marker substrate in an assay where the aldehyde product, BAL, was measured directly. The affinity values of BA for VAP-1 in rhVAP-1, hUCM, hAM, and hP were very similar to the previously reported values (Claud et al., 2002), and its rates of deamination were highly correlated with both the oxidative deamination of emixustat and the VAP-1 levels measured by ELISA in individual human tissue sources of VAP-1 (Fig. 4; Table 3). The variability of in vitro oxidative deamination in elderly hAM (8.8- to 17-fold) is comparable to the in vivo variability of emixustat plasma AUCs in AMD patients within each dose cohort (4- to 6-fold). This suggests that clinical variability in emixustat exposure is explained in part by interindividual variability in the expression of VAP1 and reinforces the use of these membranes as an in vitro model for assessing the potential impact of inhibitors of VAP-1 on emixustat metabolic clearance.

In several mammalian species, including humans, VAP-1 has been found both on vascular cell membranes and in a soluble form in plasma, with both forms determined to be the same enzyme from protein sequencing and enzymology characterization (Smith et al., 1998). Soluble VAP-1 in human plasma may originate from proteolytic cleavage of the membrane-spanning N-terminal domains of membrane-bound homodimers, resulting in a circulating enzyme that retains deamination activity (Salmi and Jalkanen, 1992) or from circulating inflammatory endothelial cells that detach from sites of injury (Holmén et al., 2005). VAP-1 deamination in human plasma and tissues has been compared with other mammals yielding extreme variation in $V_{\max }$ values (10,000-fold), with less variation in $K_{M}$. Plasma BA deamination in humans has been reported to be comparatively low, with BA deamination in human tissues being higher than in tissues from rat and pig (Boomsma et al., 2000). Markedly higher BA deamination in human vascular membranes than in plasma is consistent with the findings in the current study. In addition, we previously reported that ACU-5201 was detected after 1-hour incubation in human plasma (Fitzsimmons et al., 2018), with $85 \%-96 \%$ of $\left[{ }^{14} \mathrm{C}\right]$ emixustat remaining after 1 hour in human plasma and blood. Collectively, this suggests that the membrane-bound form of VAP-1 has a larger contribution to the rapid in vivo clearance of emixustat than the soluble plasma form.

Initially, selective chemical inhibition was used in reaction phenotyping to confirm that ACU-5201 formation was inhibited by known inhibitors of VAP-1 but not those selective for MAO-A, MAO-B, or LOX (see Fig. 5). Although inhibition of VAP-1 has been investigated extensively as an anti-inflammatory therapy over the last few decades (Kinemuchi et al., 2004; Salter-Cid et al., 2005; Dunkel et al., 2008), clinical drug interactions have yet to be observed since few drugs are eliminated by this pathway. In the absence of established clinical observations, an in vitro screening approach was undertaken for emixustat oxidative deamination. Therapeutic agents were surveyed in the literature and screened in pooled sources of human VAP-1 to investigate the potential for inhibitory DDIs. Inhibitors and each 
substrate (emixustat or BA) were coincubated prior to adding the appropriately diluted human VAP-1 sources to start the reactions. The sensitivity of the two substrates to inhibition differed by as much as 5-fold in $\mathrm{IC}_{50}$ values for guanabenz in hAM. A similar difference was seen for hydralazine against BA deamination in hAM relative to rhVAP1. The homodimeric structure of VAP-1 may contribute to these differences since both cooperativity and half-site reactivity have been observed for VAP-1 (Klema and Wilmot, 2012). Additionally, formation of imine Schiff bases with primary amine substrates in the active site of VAP-1 competes with the hydrazine inhibitors as the analogous hydrazone adducts. Given the greater intrinsic hydrolytic stability of the hydrazones, time-dependent processes that were not accounted for in the coincubation design may also contribute to the differential sensitivity.

Hydralazine is a hydrazine-containing drug that was a relatively potent inhibitor of emixustat and BA deamination in pooled hAM with $\mathrm{IC}_{50}$ values of 7.4 and $6.4 \mu \mathrm{M}$, respectively. At this time, the ratio of the plasma levels of VAP-1 inhibitors relative to their in vitro inhibition parameters has yet to be explored as quantitatively meaningful in assessing the likelihood of a clinically relevant drug interaction. The potential for hydralazine to have an impact on emixustat plasma exposures via VAP-1 inhibition remains valuable in identifying potential extrinsic factors that may impact therapeutic response to emixustat. Summation of the in vitro inhibition results presented here suggests that VAP-1 is relatively less sensitive to semicarbazide inhibition.

In summary, the data presented here show that VAP-1 is predominantly responsible for emixustat oxidative deamination in human vascular membranes (hUCM and hAM) and hP, with much higher rates in the membranes. Chemical inhibition and recombinant human enzyme strategies were effective in establishing the role of VAP-1 in emixustat metabolism and offer a simple path for drug-discovery candidates comprising primary amine to carboxylic acid biotransformations. The three major metabolites found in plasma are formed rapidly and eliminated in a formation-rate-limited fashion and can be formed sequentially in vitro with additional assay components for aldehyde dehydrogenase and P450. The rates of VAP-1 marker substrate activity and emixustat deamination were highly variable in a population of 20 elderly individuals, yet they were highly correlated with each other and VAP-1 levels measured by ELISA. Variability observed in the in vitro systems tested was similar to that observed in a clinical PK study. Most potential comedications tested did not inhibit emixustat oxidative deamination in vitro, with the exception of the vasodilators hydralazine and guanabenz. Last, clinically relevant inhibition of VAP-1 by emixustat seems unlikely since its plasma concentrations are over 10,000-fold less than the calculated $\mathrm{K}_{\mathrm{i}}$.

\section{Acknowledgments}

We thank Susan Henry (Acucela, VAP-1 ELISA), Vladimir Kuksa (Acucela, synthetic standard preparation and characterization), Lou Anne McKown (Acucela, synthetic inhibitor preparation and in vivo rat inhibitor PK analysis), Lauren Benoit (Skills Alliance, VAP-1 marker substrate assay and emixustat deamination assay), Yan Wang (Skills Alliance, VAP-1 ELISA and marker substrate assay), Galen Hall (Oregon State University, emixustat sequential metabolism), Michael Fitzsimmons (Covance, plasma stability, rat portal vein ADME, MAO, and hLM assays), and Inger M. Darling (Cognigen, PK analysis).

\section{Authorship Contributions}

Participated in research design: Reid, Eyre, Podoll.

Conducted experiments: Reid, Podoll.

Performed data analysis: Reid, Podoll.

Wrote or contributed to the writing of the manuscript: Reid, Eyre, Podoll.

\section{References}

Awh CC (2016) An oral drug for treatment of AMD? Retina Today July/August, pp 83-84. Barrand MA and Callingham BA (1985) The interaction of hydralazine with a semicarbazidesensitive amine oxidase in brown adipose tissue of the rat. Its use as a radioactive ligand for the enzyme. Biochem J 232:415-423.

Bavik C, Henry SH, Zhang Y, Mitts K, McGinn T, Budzynski E, Pashko A, Lieu KL, Zhong S, Blumberg B, et al. (2015) Visual cycle modulation as an approach toward preservation of retinal integrity. PLoS One 10:e124940.

Boomsma F, van den Meiracker A, Man in 't Veld A, and Schalekamp M (1995) Contrasting effects of peripheral decarboxylase inhibitors on plasma activity of aromatic-L-amino acid decarboxylase and semicarbazide-sensitive amine oxidase in Parkinson's disease. Life Sci 57: $1753-1759$.

Boomsma F, van Dijk J, Bhaggoe UM, Bouhuizen AM, and van den Meiracker AH (2000) Variation in semicarbazide-sensitive amine oxidase activity in plasma and tissues of mammals. Comp Biochem Physiol C Toxicol Pharmacol 126:69-78.

Claud P, Artur Y, and Laine R (2002) In vitro metabolism of tresperimus by human vascular semicarbazide-sensitive amine oxidase. Drug Metab Dispos 30:747-755.

Claud P, Padovani P, Guichard J-P, Artur Y, and Lainé R (2001) Involvement of semicarbazidesensitive amine oxidase in tresperimus metabolism in human and in rat. Drug Metab Dispos 29: 735-741.

Constantino L, Paixão P, Moreira R, Portela MJ, Do Rosario VE, and Iley J (1999) Metabolism of primaquine by liver homogenate fractions. Evidence for monoamine oxidase and cytochrome P450 involvement in the oxidative deamination of primaquine to carboxyprimaquine. Exp Toxicol Pathol 51:299-303.

Dugel PU, Novack RL, Csaky KG, Richmond PR, Birch DG, and Kubota R (2015) Phase II, randomized, placebo-controlled, 90-day study of emixustat HCL in geographic atrophy associated with dry age-eelated macular degeneration. Retina 35:1173-1183.

Dunkel P, Gelain A, Barlocco D, Haider N, Gyires K, Sperlágh B, Magyar K, Maccioni E, Fadda A, and Mátyus P (2008) Semicarbazide-sensitive amine oxidase/vascular adhesion protein 1: recent developments concerning substrates and inhibitors of a promising therapeutic target. Curr Med Chem 15:1827-1839.

Food and Drug Administration (2016) Drug Development and Drug Interactions: Table of Substrates, Inhibitors and Inducers. Food and Drug Administration, Silver Spring, MD.

Fitzsimmons ME, Sun G, Kuksa V, and Reid MJ (2018) Disposition, profiling and identification of emixustat and its metabolites in humans. Xenobiotica 48:592-604.

Frischer H, Mellovitz RL, Ahmad T, and Nora MV (1991) The conversion of primaquine into primaquine-aldehyde, primaquine-alcohol, and carboxyprimaquine, a major plasma metabolite. $J$ Lab Clin Med 117:468-476.

Garpenstrand H, Ekblom J, Bäcklund LB, Oreland L, and Rosenqvist U (1999) Elevated plasma semicarbazide-sensitive amine oxidase (SSAO) activity in type 2 diabetes mellitus complicated by retinopathy. Diabet Med 16:514-521.

Gower NJD, Barry RJ, Edmunds MR, Titcomb LC, and Denniston AK (2016) Drug discovery in ophthalmology: past success, present challenges, and future opportunities. $B M C$ Ophthalmol 16:11.

Heuts DP, Gummadova JO, Pang J, Rigby SE, and Scrutton NS (2011) Reaction of vascular adhesion protein-1 (VAP-1) with primary amines: mechanistic insights from isotope effects and quantitative structure-activity relationships. J Biol Chem 286:29584-29593.

Holmén C, Elsheikh E, Stenvinkel P, Qureshi AR, Pettersson E, Jalkanen S, and SumitranHolgersson S (2005) Circulating inflammatory endothelial cells contribute to endothelial progenitor cell dysfunction in patients with vasculitis and kidney involvement. J Am Soc Nephrol 16:3110-3120.

Holt A, Alton G, Scaman CH, Loppnow GR, Szpacenko A, Svendsen I, and Palcic MM (1998) Identification of the quinone cofactor in mammalian semicarbazide-sensitive amine oxidase. Biochemistry 37:4946-4957.

Holt A, Sharman DF, Baker GB, and Palcic MM (1997) A continuous spectrophotometric assay for monoamine oxidase and related enzymes in tissue homogenates. Anal Biochem 244:384-392.

Kinemuchi H, Sugimoto H, Obata T, Satoh N, and Ueda S (2004) Selective inhibitors of membrane-bound semicarbazide-sensitive amine oxidase (SSAO) activity in mammalian tissues. Neurotoxicology 25:325-335.

Klema VJ and Wilmot CM (2012) The role of protein crystallography in defining the mechanisms of biogenesis and catalysis in copper amine oxidase. Int J Mol Sci 13:5375-5405.

Kubota R, Al-Fayoumi S, Mallikaarjun S, Patil S, Bavik C, and Chandler JW (2014) Phase 1, doseranging study of emixustat hydrochloride (ACU-4429), a novel visual cycle modulator, in healthy volunteers. Retina 34:603-609.

Kubota R, Boman NL, David R, Mallikaarjun S, Patil S, and Birch D (2012) Safety and effect on rod function of ACU-4429, a novel small-molecule visual cycle modulator. Retina 32:183-188.

Kurkijärvi R, Yegutkin GG, Gunson BK, Jalkanen S, Salmi M, and Adams DH (2000) Circulating soluble vascular adhesion protein 1 accounts for the increased serum monoamine oxidase activity in chronic liver disease. Gastroenterology 119:1096-1103.

Lalor PF, Edwards S, McNab G, Salmi M, Jalkanen S, and Adams DH (2002) Vascular adhesion protein-1 mediates adhesion and transmigration of lymphocytes on human hepatic endothelial cells. J Immunol 169:983-992.

Lizcano JM, Tipton KF, and Unzeta M (1998) Purification and characterization of membranebound semicarbazide-sensitive amine oxidase (SSAO) from bovine lung. Biochem J 331 69-78.

Marchitti SA, Brocker C, Stagos D, and Vasiliou V (2008) Non-P450 aldehyde oxidizing enzymes: the aldehyde dehydrogenase superfamily. Expert Opin Drug Metab Toxicol 4:697-720.

Mercier N, Kakou A, Challande P, Lacolley P, and Osborne-Pellegrin M (2009) Comparison of the effects of semicarbazide and beta-aminopropionitrile on the arterial extracellular matrix in the Brown Norway rat. Toxicol Appl Pharmacol 239:258-267.

Mihaly GW, Ward SA, Edwards G, Orme ML, and Breckenridge AM (1984) Pharmacokinetics of primaquine in man: identification of the carboxylic acid derivative as a major plasma metabolite. Br J Clin Pharmacol 17:441-446.

Olivieri A and Tipton K (2011) Inhibition of bovine plasma semicarbazide-sensitive amine oxidase by caffeine. J Biochem Mol Toxicol 25:26-27.

Pannecoeck R, Serruys D, Benmeridja L, Delanghe JR, van Geel N, Speeckaert R, and Speeckaer MM (2015) Vascular adhesion protein-1: role in human pathology and application as a biomarker. Crit Rev Clin Lab Sci 52:284-300.

Podoll T, Al-Fayoumi S, Eyre R, Austin E, Prescott E, Sun G, Orme M, and Kubota R (2013) Comparative nonclinical ocular tissue distribution of the visual cycle modulator (VCM) emixustat in rats, beagle dogs and cynomolgus monkeys. Toxicologist 132:107. 
Podoll T, Geisler L, Parys MV, Hanson G, and Reid MJ (2018) Validation and reproducibility of an LC-MS/MS method for emixustat and its three deaminated metabolites in human plasma. Bioanalysis 10:1803-1817.

Precious E, Gunn CE, and Lyles GA (1988) Deamination of methylamine by semicarbazidesensitive amine oxidase in human umbilical artery and rat aorta. Biochem Pharmacol 37: 707-713.

Pybus BS, Sousa JC, Jin X, Ferguson JA, Christian RE, Barnhart R, Vuong C, Sciotti RJ, Reichard GA, Kozar MP, et al. (2012) CYP450 phenotyping and accurate mass identification of metabolites of the 8-aminoquinoline, anti-malarial drug primaquine. Malar J 11:259.

Salmi M and Jalkanen S (1992) A 90-kilodalton endothelial cell molecule mediating lymphocyte binding in humans. Science 257:1407-1409.

Salmi M, Kalimo K, and Jalkanen S (1993) Induction and function of vascular adhesion protein-1 at sites of inflammation. J Exp Med 178:2255-2260.

Salter-Cid LM, Wang E, O'Rourke AM, Miller A, Gao H, Huang L, Garcia A, and Linnik MD (2005) Anti-inflammatory effects of inhibiting the amine oxidase activity of semicarbazidesensitive amine oxidase. J Pharmacol Exp Ther 315:553-562.

Schwelberger HG (2007) The origin of mammalian plasma amine oxidases. J Neural Transm (Vienna) 114:757-762.
Smith DJ, Salmi M, Bono P, Hellman J, Leu T, and Jalkanen S (1998) Cloning of vascular adhesion protein 1 reveals a novel multifunctional adhesion molecule. J Exp Med 188:17-27. Smith PK, Krohn RI, Hermanson GT, Mallia AK, Gartner FH, Provenzano MD, Fujimoto EK Goeke NM, Olson BJ, and Klenk DC (1985) Measurement of protein using bicinchoninic acid Anal Biochem 150:76-85.

Stopher DA, Beresford AP, Macrae PV, and Humphrey MJ (1988) The metabolism and pharmacokinetics of amlodipine in humans and animals. J Cardiovasc Pharmacol 12 (Suppl 7): S55-S59.

van Dijk J, Boomsma F, Alberts G, Man in 't Veld AJ, and Schalekamp MA (1995) Determination of semicarbazide-sensitive amine oxidase activity in human plasma by high-performance liquid chromatography with fluorimetric detection. J Chromatogr B Biomed Appl 663:43-50.

Wibo M, Duong AT, and Godfraind T (1980) Subcellular location of semicarbazide-sensitive amine oxidase in rat aorta. Eur J Biochem 112:87-94.

Address correspondence to: Dr. Michael J. Reid, Acucela Inc., 818 Stewart Street, Seattle, WA 98101. E-mail: mjr33_33@yahoo.com 